\title{
Adar-mediated A-to-I editing is required for establishment of embryonic body axes in zebrafish
}

Katarzyna Niescierowicz ${ }^{1 *}$, Leszek Pryszcz ${ }^{1 *}$, Cristina Navarrete ${ }^{1 *}$, Eugeniusz Tralle ${ }^{1}$, Marta Elżbieta Kasprzyk ${ }^{1}$, Karim Abu Nahia ${ }^{1}$, Katarzyna Misztal ${ }^{1}$, Matthias Bochtler ${ }^{1 \dagger}$, Cecilia Winata ${ }^{1 \dagger}$

${ }^{1}$ International Institute of Molecular and Cell Biology in Warsaw, Poland

*equal contribution

†corresponding authors: mbochtler@iimcb.gov.pl, cwinata@iimcb.gov.pl

Keywords: ADAR, A-to-I editing, zebrafish, anteroposterior, dorsoventral patterning 


\begin{abstract}
Adenosine deaminases (ADARs) catalyze the deamination of adenosine to inosine, also known as A-to-I editing, in RNA. Although A-to-I editing occurs widely across animals, and is well studied, new biological roles are still being discovered. Here, we study the role of A-to-I editing in early zebrafish development. We demonstrate that Adar, the zebrafish orthologue of mammalian ADAR1, is essential for establishing the antero-posterior and dorso-ventral axes and patterning. Genome-wide editing discovery revealed pervasive editing in maternal and the earliest zygotic transcripts, the majority of which occurred in the 3'-UTR. Interestingly, transcripts implicated in gastrulation as well as dorsoventral and antero-posterior patterning were found to contain multiple editing sites. Adar knockdown or overexpression affected gene expression and global editing patterns at $\mathbf{1 2} \mathrm{hpf}$, but not earlier. Our study established that RNA editing by Adar is necessary for the earliest steps of embryonic patterning along the zebrafish antero-posterior and dorso-ventral axes.
\end{abstract}

\title{
Introduction
}

RNA editing is a phenomenon of post-transcriptional alteration of transcript primary sequence $^{[1]}$. Its most common form is the A-to-I editing, in which adenosine $(A)$ at the C6 position is deaminated, giving rise to an inosine $(\mathrm{I}){ }^{[2,3]}$. As inosine pairs like guanine with tRNAs, A-to-I editing has the potential to alter the coding capacity of mRNAs, in some cases with drastic biological consequences ${ }^{[4,5]}$. However, because of a prevalence of A-to-I editing in double-stranded RNA regions, most editing events do not affect the coding capacity of the genome.

A-to-I editing occurs widely in animals, from the earliest-diverging eumetazoan phyla to man ${ }^{[6,7]}$. In vertebrates ${ }^{[8]}$ and invertebrates ${ }^{[9]}$, A-to-I editing prevents autoimmunity that is triggered by endogenous dsRNA ${ }^{[10-12]}$. Another recurring theme of A-to-I editing is its role in the brain. In the mouse and zebrafish, editing of the GluR2 transcript is important for normal development of the nervous system ${ }^{[5,13,14]}$. In the fruit fly, perturbed A-to-I editing causes behavioral phenotypes ${ }^{[15]}$. In certain ant species, it determines castespecific behavior ${ }^{[16]}$. In the squid nervous system, extensive A-to-I editing is more prevalent in the giant axon system compared to the cell body, indicating region-specific editing within a neuron cell ${ }^{[17,18]}$. In human and mouse, A-to-I editing contributes to germline integrity, by preventing the spread of Alu ${ }^{[19-21]}$ and SINE elements [22], respectively. Higher editing prevalence in zebrafish testis and ovary compared to other organs ${ }^{[23]}$ may hint to a role of A-to-I editing for germline integrity in non-mammalian 
vertebrates. However, to our knowledge, there is no evidence for this role in invertebrates yet.

A-to-I editing is catalyzed by adenosine deaminases (ADARs) ${ }^{[3]}$. Most vertebrates have three paralogues that have arisen prior to vertebrate radiation, and can therefore be expected to have biochemically similar functions and substrate preferences ${ }^{[3,24]}$. Apart from a C-terminal deaminase domain, all ADARs have at least two, and in some cases three double-stranded DNA (dsDNA) binding domains. ADAR1 additionally has a ZDNA binding domain (ZBD) at the amino-terminal end of the protein that is missing from the other deaminases ${ }^{[25]}$. Among the ADAR paralogues, only ADAR1 and ADAR2 are active, whereas ADAR3 has an inactive catalytic domain and appears to fulfill its biological role in the absence of catalytic activity ${ }^{[26,27]}$.

In mammals, ADAR1 and ADAR2 are widely expressed, whereas ADAR3 is only expressed at low levels in the brain ${ }^{[3]}$, particularly in the amygdala and hypothalamus [28]. The majority of A-to-I editing is performed by ADAR1 and prevents dsRNA mediated autoimmunity ${ }^{[11]}$. ADAR1/Adar1 occurs as two isoforms, known as p110 and p150 [29]. Both share the deaminase domains, the dsRNA-binding domains, and one Z DNAbinding domain. Additionally, p150 possesses a second Z DNA-binding domain at the $\mathrm{N}$-terminal end ${ }^{[30]}$. Mice with a homozygous knockout of either both isoforms or only the p150 isoform cannot complete embryonic development. They die between embryonic day 11.5 and 12.5 from failed erythropoiesis and fetal liver disintegration [31,32], presumably due to stress in these cells ${ }^{[33]}$. In contrast to Adar1 null mice, Adar2 mutant mice can complete embryonic development, but die subsequently from seizures, within three weeks of birth ${ }^{[5]}$. Remarkably, this phenotype depends on a single editing event in the GluA2 AMPA receptor transcript. Even though multiple transcripts in the brain are edited ${ }^{[4]}$, a point mutation in the GluA2 transcript suffices to suppress the Adar2 null phenotype. In humans, ADAR2 is associated with epilepsy, neurodegeneration, and autism $^{[34]}$.

In zebrafish, almost all data about A-to-I editing are descriptive. In contrast to most other vertebrates, which have three ADAR paralogues, zebrafish have four, due to a duplication of the ADAR2 ortholog into adarb1a and adarb1b. In the following, we use the official zebrafish nomenclature: adar for adar1, adarb1a for adar2a, adarb1b for $a d a r 2 b$, and adarb2 for adar3. Transcripts for adar, and to a lesser extent adarb1b, are highly abundant during the first few hours of development. Transcripts of the other adenosine deaminase genes are scarce or absent during the first few hours. Although they are eventually expressed later on, the transcript levels remain lower than those of adar and adarb1b throughout development. Sequencing data suggests that editing in transcripts from repetitive genomic elements is pervasive in the first few hours of 
development, before the maternal to zygotic transition, and much less pronounced in later developmental stages ${ }^{[23]}$. Editing in coding regions of genes sets in only later, roughly one day after fertilization. In adult organs, adar was most highly expressed in testis and heart, whereas adarb1a was highly expressed in heart and brain. Overall Ato-I editing was most pervasive in testes and ovaries ${ }^{[23]}$. In contrast to the detailed information about the occurrence of editing, very little is known about functional consequences. It is known from earlier work that editing of GluR2 is conserved in zebrafish and is essential for normal development of the nervous system and cranial neural crest cells ${ }^{[14]}$. However, it is unclear how conserved the role of A-to-I editing is otherwise, particularly for the early stages of development that differ greatly between mammals and zebrafish.

Here, we explore the role of A-to-I editing in early zebrafish embryos, focusing on the most highly expressed adar. Knockdown and overexpression experiments revealed that maternal adar is essential for zebrafish development, particularly during the earliest steps of antero-posterior and dorso-ventral patterning, and that this function is dependent on an intact deaminase domain. Transcriptome analysis uncovered prevalent A-to-I RNA editing during early embryogenesis, which affects transcripts known to play a role in gastrulation as well as dorso-ventral and antero-posterior patterning. adar knockout experiments further demonstrated later roles for the gene that could not be observed in the knockdown experiments because of the earlier lethality.

\section{Results}

\section{The A-to-I RNA editing enzyme Adar and Adarb1b are expressed in the developing zebrafish embryo}

To determine whether A-to-I editing activity exists during embryonic development, we revisited our transcriptome data ${ }^{[35]}$ to check whether the enzymes responsible for A-to-I editing were expressed. In agreement with other recently published data [23], we detected transcripts of at least two deaminase paralogs, adar and adarb1b, from egg to $5.3 \mathrm{hpf}$ (Fig. 1A). Transcripts of these two paralogs were present both maternally as well as zygotically, with adar being more abundant (more than 4-fold compared to adarb1b at each developmental stage). Moreover, we found that transcripts of both paralogs were consistently associated with polysome, starting from the egg stage up to $5.3 \mathrm{hpf}$ (Fig. 1B). This finding suggests that these transcripts are constantly undergoing translation at developmental stages preceding and after the activation of zygotic genome ${ }^{[35]}$. The observation that both paralogs were already expressed, and their transcripts associated with polysomes at egg stage suggests that RNA editing events occur prior to fertilization and may be crucial for early development. Interestingly, a 
substantial increase in adar expression occurs after the mid-blastula transition (MBT), suggesting that the role of this gene extends beyond the period of transcriptional silence in early embryogenesis (Fig. 1A). At larval stage, the expression of both gene paralogs was not spatially restricted although more abundant in the nervous system of the developing embryo as shown by whole mount in situ hybridization of adar (Fig.1C, D) and adarb1b (Fig. 1E, F) in 24 hpf zebrafish embryos.

\section{The A-to-I editing activity of Adar is required for early embryonic patterning}

To investigate whether Adar plays a biological role in early zebrafish development, we used morpholino oligonucleotides (MO) to knock-down Adar, the highest expressed among all zebrafish ADAR paralogs (Fig. 1A). Adar MO-injected embryos developed a range of phenotypes which is initially evident at gastrulation and subsequently observed to affect posterior body axis by $24 \mathrm{hpf}$ (Fig. 2A-C, H). The most severe phenotypes manifested in a lack of almost all posterior structures and crooked body axis (Fig. 2C). In addition, the notochord in morphant embryos was disorganized, with unevenly shaped and distributed vacuoles instead of the neat "stack of coins" arrangement in wild-type (Fig. 2B, C). This abnormal MO phenotype was dose-dependent and could be rescued with the wild-type adar mRNA, in which an increased percentage of embryos appeared normal or exhibited mild phenotype with proper body axis organization and tail length (Fig. 2D, H; Supplementary Fig. 1). On the other hand, knockdown with up to 2ng of MO against Adarb1b, which was expressed at a lower level in the early embryo, did not result in any observable phenotype (Supplementary Fig. 1). To verify whether the biological role of Adar depends on its RNA editing activity, we generated adar mRNA E1030A designed after a similar construct in mammals ${ }^{[36]}$, in which the deaminase domain was mutated (Supplementary Figure 1). The E1030A mRNA was unable to rescue MO-induced phenotype in developing embryos, resulting in comparable number of abnormally developed embryos with severe posterior axis defects to that of MO-injected larvae (Fig. 2E, H). This suggests that a functional deaminase domain, catalyzing the A-to-I editing in dsRNA, is essential for early embryonic development. These results strongly suggest that the A-to-I editing activity of Adar is necessary for the specification of early embryonic axes.

Whereas Adar MO affected the development of the posterior part of the body, adar mRNA overexpression caused significant abnormalities of the anterior part, which include anomalous eye development, most often manifested in cyclopia, deformed cranium, and reduced or absent brain compartment, particularly at the anteriormost region (Fig. 2F, G, I). These defects were most prominently observed with $50 \mathrm{pg}$ injection of mRNA. The effect of adar mRNA overexpression was dose-dependent with a $64 \%$ mortality for the $100 \mathrm{pg}$ of injected mRNA, 9\% for $50 \mathrm{pg}$ and $7 \%$ for $25 \mathrm{pg}$ 
(compared to wt which had $5 \%$ mortality). Collectively, our results show that Adar plays a key role in the earliest steps of embryonic patterning.

In order to characterize the observed morphological defects of Adar loss- and gain- of function in more detail, we assessed the expression of several marker genes indicating various embryonic structures. The most prominent phenotype of Adar disruption is that of the development of structures along the anteroposterior axis. In order to characterize the effect of Adar loss- or gain-of function on the development of anterior embryonic structures, we utilized the expression of pax 6 and $t b x 2 b$ to collectively indicate, among which, the forebrain, midbrain, and hindbrain, anterior spinal cord, optic vesicle, and otic vesicle (Fig. 3A, D, G, J) ${ }^{[37]}{ }^{[38]}$. In adar morphants where morphological defects were predominantly observed in the posterior region, these anterior structures were preserved and appeared similar to wild-type in terms of their size and organization (Fig. $3 \mathrm{~B}, \mathrm{E}, \mathrm{H}, \mathrm{K})$. On the contrary, anteriormost brain regions and optic vesicle were indistinguishable in embryos overexpressing adar (Fig. 3C, F, I, L). In particular, pax6 expression shows that the diencephalon and telencephalon expression domain were unrecognizable in adar-overexpressing embryos (Fig. 3C, F). Moreover, tbx2b expression domains of telencephalon, left and right optic vesicles, and the epiphysis appeared as a fused region, whereas the more posterior expression domains of the trigeminal ganglion and otic vesicles appeared less affected although hypomorphic compared to control (Fig. 3I, L). While the eye field is derived from the anterior neural plate and therefore arose as a consequence of antero-posterior patterning ${ }^{[39]}$, its failure to split in the midline (cyclopia) is a hallmark of defect in the convergent-extension movement during gastrulation which is dependent on the establishment of the dorsoventral axis ${ }^{[40,41]}$. The observed phenotype of adar overexpression therefore suggests that proper establishment of these two body axes was affected.

To examine the defects in posterior structures, we used as reporter genes tbxta and shhb which mark the notochord and floor plate neurons of the spinal cord, respectively (Fig. 3M, P). Similar to the anterior markers, shha and tbxta were expressed in the case of both adar loss- or gain-of-function, suggesting that the neural and midline cell identities were preserved (Fig. 3N, O, Q). However, the body axis including the notochord and spinal cord were crooked in adar morphants as well as in adar overexpressing embryos albeit to a lesser extent in the latter (Fig. 3N, O). Importantly, loss- or gain-of-function of adar do not appear to affect the specification of cell identity as evidenced by the preserved expression of pax6, tbx $2 b$, shha, and tbxta. Rather, $a$ more pronounced effect is seen on the gross anteroposterior and dorsoventral patterning of the embryo. Collectively, these observations suggest that Adar-mediated A-to-I editing may be involved in the patterning along the two early embryonic axes of the zebrafish. 


\section{A-to-I RNA editing is prevalent in maternal and early zygotic transcripts during normal embryogenesis}

The effects of Adar disruption on embryonic patterning led us to ask to what extent A-toI editing occurs in early embryogenesis, and whether transcripts of genes involved in this process were specifically affected. To profile global A-to-I RNA editing in zebrafish we sequenced a trio of wild-type sample of both parental genomes and the transcriptome of their offspring at three developmental stages: $1.5 \mathrm{hpf}$ (16-cells, preMBT), 3.5 hpf (high, MBT) and $5.3 \mathrm{hpf}$ (50\% epiboly, post-MBT) (Fig. 4A). Comparison of zygotic transcriptomes with the genomic sequence of parents allowed us to pinpoint RNA editing events by identifying mismatches between the genomic and transcriptomic reads. As Inosine (I) is structurally similar to a $G$ due to the presence of the 6-oxo group, reverse transcriptase incorporates a $C$ in the corresponding position during RNAseq library synthesis; thus, in the original transcript strand, a $G$ is inferred. Therefore an A-to-l editing event can be identified as an A-G mismatch between the parental genome and the corresponding embryonic transcript. Since the RNA-seq was unstranded, the same applies as well to a T-C mismatch. Strikingly, our analyses revealed a disproportionate enrichment of A-G and T-C mismatches compared to other possible base mismatches which could occur stochastically at all three developmental stages (Fig. 4B). This strongly suggests the presence of A-to-I RNA editing in maternally deposited as well as in zygotic transcripts. Altogether, we identified 44,007 RNA editing sites: 11,374 of which were shared by all three samples, 6352 between $1.5 \mathrm{hpf}$ and 3.5 hpf, 1393 between $1.5 \mathrm{hpf}$ and $5.3 \mathrm{hpf}$, and 1,564 between $3.5 \mathrm{hpf}$ and $5.3 \mathrm{hpf}$. Apart from this, 6117, 5686 and 11,521 were specific to $1.5 \mathrm{hpf}, 3.5 \mathrm{hpf}$ and $5.3 \mathrm{hpf}$, respectively (Fig. 4C, Supplementary Table 1). These stage-specific editing events indicate differential patterns of RNA editing throughout early embryogenesis and suggest that this process may play a role in embryogenesis. Interestingly, merely $2 \%$ of RNA editing sites occurred within coding sequences (CDS), while the majority ( $38 \%$ in $1.5 \mathrm{hpf}$ and $3.5 \mathrm{hpf}$ and $27 \%$ in $5.3 \mathrm{hpf}$ ) occurred in 3'-UTR regions (Fig. 4D, Supplementary Table 1). A large fraction of editing was assigned as 'genic_other' due to overlap between intron/exon/UTRs from multiple transcripts. Finally, $17 \%$ of editing events in each stage were detected in 'intergenic sequence', but this may be due to unannotated transcripts. The low abundance of A-to-I editing present in the coding regions (CDS), none of which resulting in missense mutations, ruled out the possibility that editing functions through expanding the coding repertoire of expressed transcripts by altering amino acid composition. We also observed plenty of editing occurring in DNA repeat regions with a notable increase in editing frequency in retrotransposons (LTR, LINE, and SINE) in $5.3 \mathrm{hpf}$ compared to earlier developmental stages (Fig. 4E, Supplementary Table 2). 
To assess the penetrance of A-to-I editing on individual RNA molecules, we calculated the occurrence of editing events in RNA-seq reads. Based on alignment of 75bp reads, we observed a substantial number of reads with no editing at all for a given region (Fig. $4 \mathrm{~F})$. Interestingly, the number of RNA editing sites in a single read never exceeds $50 \%$ of the total number of editing events identified in the given transcript region (Fig. 4F, Supplementary Table 3). This suggests that A-to-I editing rarely occurs at all possible editing sites in a given RNA molecule, although sequencing with much longer reads is required to precisely determine the true penetrance. We then ask whether RNA editing events were associated with translation efficiency and/or RNA stability. Using our previously published polysome profiling dataset to determine translation rate ${ }^{[35]}$, we found that transcripts undergoing more active translation tend to be less edited than those not associated with polysome (Supplementary Fig. 2). Interestingly, this is true only for editing events detected at post-MBT stages (3.5 hpf and $5.3 \mathrm{hpf}$ ), but not for pre-MBT (1.5 hpf). With regards to RNA stability, no obvious difference was observed in expression levels between low (1-9 sites) and high (more than 10 sites) edited transcripts when $1.5 \mathrm{hpf}$ and $3.5 \mathrm{hpf}$ stages were compared. However, highly edited transcripts undergo much lower expression change between $1.5 \mathrm{hpf}$ and $5.3 \mathrm{hpf}$ (Supplementary Fig. 3).

We sought to identify which genes were subject to A-to-I editing at each developmental stage. We identified 639, 634, and 562 genes having at least two editing sites at any position within their transcript at $1.5 \mathrm{hpf}, 3.5 \mathrm{hpf}$, and $5.3 \mathrm{hpf}$ respectively (Supplementary Table 4). Among these, 9, 9, and 10 genes had editing sites occurring in both 3'UTR and coding sequence at each respective stage. Interestingly, although no developmentally relevant GO terms were found to be significantly enriched among edited genes (Supplementary Table 5), we found several key genes known to be implicated in anteroposterior and dorsoventral patterning (Fig. 5). Of note, members of the Wnt signaling pathway were found to be edited. Several Frizzled receptors of Wnt signaling, $f z d 3 b, f z d 5$, and $f z d 8 b$ were edited at both maternal stages, while $f z d 7 b$ were edited at $5.3 \mathrm{hpf}$. The Wnt downstream effector $t c f 7 / 1 \mathrm{~b}$ were found to be consistently edited at all three stages. We also found two members of the FGF signaling pathway, fgfr1a and ext/3, consistently edited at all three stages observed. Interestingly, fgfr1a is one of the highest edited transcripts, containing 99 edited sites in both its coding sequence and 3'-UTR. Mutation of FGFR1 in humans is associated with holoprosencephaly ${ }^{[42]}$ which is reminiscent of the observed adar overexpression phenotype. Other genes which were consistently edited throughout the three stages include furina which plays a role in craniofacial development ${ }^{[43]}$ and two genes involved in gastrulation, dusp4 and ezrb ${ }^{[44,45]}$. That transcripts of these genes were found to be 
edited throughout maternal and early zygotic stages suggests the role of A-to-I editing in regulating multiple aspects of anteroposterior and dorsoventral patterning.

\section{Maternal-Zygotic Adar loss of function affects dorsoventral patterning without observable changes in global A-to-I editing patterns}

To uncover the role of Adar at the molecular level, we performed transcriptome sequencing of wild-type, Adar MO knockdown (KD) and Adar overexpressing (OE) embryos at 128-cells (before ZGA), $5.3 \mathrm{hpf}$ (after ZGA), and $12 \mathrm{hpf}$ (when embryonic patterning is established). We hypothesized that alterations in the level of adar transcripts may evoke consequential differences in mRNA editing and/or changes in the expression of potential target genes. To this end, we performed comparative transcriptome analysis of wt vs $\mathrm{OE}$ and wt vs KD in each developmental stage. Surprisingly, no substantial changes in global A-to-I editing levels were found in KD and OE conditions at 128-cell and 5.3 hpf stages (Supplementary Fig. 4A). Moreover, during these two stages, samples clustered according to developmental stage (Supplementary Fig. 4B) and no substantial changes were observed in global gene expression profile $(p<0.05$; Supplementary Fig. $4 C)$. Only 539 sites have a slightly elevated editing level in $\mathrm{OE}$ than in KD in both developmental stages (Supplementary Table 6).

In contrast, at $12 \mathrm{hpf}$, a more noticeable change in both global editing pattern and gene expression profile were observed between control and Adar gain- or loss-of-function at 12 hpf (Fig. 6A; Supplementary Fig. 5). Unlike at earlier stages, samples clustered according to conditions rather than developmental stage (Fig. 6B). Interestingly, in all three replicates, Adar KD caused a modest but more noticeable change in editing frequency compared to the earlier stages (Supplementary Fig. 5). Moreover, at $12 \mathrm{hpf}$, we observed 827 and 5054 genes differentially expressed in Adar KD and Adar OE respectively, compared to control ( $p<0.05$; Supplementary Table 7$)$. GO analysis (Supplementary Table 8) revealed that Adar KD generally caused the upregulation of genes regulating epiboly (nanog, cacnb4b), gastrulation (bc/2/10, mylipa), and ectoderm development (pou5f3, cdh1), while concurrently causing downregulation of those implicated in convergent-extension (wnt11, creb1a, ppp1cb) and mesoderm development (tbx16, hes6, her7, mcdh2, myf5, msgn1). On the other hand, Adar OE resulted in the upregulation of genes involved in the development of mesodermal structures (myf6, bves, tcf21, tbx5a, apln, mef2aa), while downregulating genes regulating epiboly (chuk, epcam, mapkapk2a, cldne), dorsoventral pattern formation (sox11b, dusp6, ved, vox, bambia, acvr1ba, ctnnb2), and brain development. Interestingly, out of the 383 genes common between the two conditions, 283 commonly downregulated genes in both Adar KD and OE included genes known for their role in anterior-posterior as well as dorsoventral patterning (Fig. 6C; Supplementary Table 7), 
such as $c d x 4^{[46]}, s z l$ and ved required for DV patterning ${ }^{[47,48]}$, and several hox genes [49]. This suggests that both Adar KD and OE may act on factors with downstream consequence of suppression of these axis-regulating genes.

As Adar gain- and loss-of-function resulted in almost opposite defects in anteriorposterior and dorso-ventral patterning, we compared the transcriptome from the two conditions and found 1218 differentially expressed genes (Supplementary Table 7). Genes regulating the development of mesodermal structures (bmpr2a, emilin3a, msgn1, gata1a, meis1b, etv2, pdgfra) as well as hox family genes (hoxa4a/9b, hoxb3a/5a, hoxc1a/3a/6a, hoxd4a) were upregulated in OE compared to KD (Supplementary Table 8). On the contrary, genes regulating epiboly and gastrulation (chuk, epcam, mapkapk2a, snai1a), convergent-extension (dsc2l, gpc4, tdgf1, ptpra, prmt1), and dorsal-ventral patterning (sox11b, ctnnb1, ppp4cb, tll1) were downregulated in $\mathrm{OE}$ compared to KD (Supplementary Table 8). These observations agree with the phenotypes resulting from Adar disruption which affected structures along the anteroposterior and dorsoventral axes, where an excess of Adar affected dorsal and anterior structures while Adar deficiency affected more ventral and posterior structures. Taken together, the consequences of Adar disruption at the molecular level could be observed by $12 \mathrm{hpf}$, which affected the expression of genes involved in the development of various embryonic structures. This suggests that, although no significant changes were observable in global editing levels and transcriptome up to 5.3 hpf, Adar is necessary during the crucial period of embryonic patterning, between gastrulation and $12 \mathrm{hpf}$.

\section{Zygotic Adar is not essential for early embryogenesis}

We then asked whether zygotic Adar activity is required for later events of embryogenesis. To this end, we created a zebrafish adar mutant line (adar-/-) using the CRISPR/Cas9-based gene knock-out method. A 5 bp deletion was introduced into the second exon of the adar gene, resulting in a premature stop codon and polypeptide consisting of $4 \%$ (39 aa) of the full length, functional Adar protein (917 aa) (Fig. 7A). To generate homozygous adar mutants we incrossed F1 heterozygous individuals and observed the F2 offspring. Surprisingly, no developmental phenotypes were observed in any of the offspring. We then raised these $\mathrm{F} 2$ individuals and genotyped them at approximately 3 months post-fertilization by fin clipping. Surprisingly, we found $76 \%$ heterozygous and $24 \%$ wild type fish among the 200 screened samples. None of the adult F2 individuals were homozygous for the adar-/- mutant allele (Fig. 7B). This suggests that the homozygous adar-/- knockout is not viable despite their lack of developmental abnormalities. To determine the fate of the homozygous adar-/- mutants, we genotyped F2 individuals ( 300 individuals) at $3 \mathrm{dpf}$. Interestingly, the distribution of 
homo-, heterozygous and wild-type individuals was in agreement with the Mendelian ratio (Fig. 7B), indicating that mortality of the homozygous adar-/- individuals occurred later than $3 \mathrm{dpf}$ and before adulthood. In order to pinpoint the timing of mortality, we followed the development of homozygous individuals until the point of death. The adar-/larvae did not demonstrate any observable morphological abnormalities when compared to heterozygotes or wild-type individuals. However, significant mortality was observed between second- and third-weeks post fertilization. Nearly $100 \%$ of adar-/mutants died within this period, with only one surviving until the third month post fertilization, with substantial growth impairment. To eliminate the possibility of genetic complementation ${ }^{[50]}$ causing an attenuation of the adar-/- phenotype, we analyzed mRNA levels of adar as well as three other adar family members: adarb1a, adarb1b and adarb2, in wild-type and adar-/- homozygotes. No significant changes in the mRNA levels of adar, adarb1a, adarb1b and adarb2 mRNAs were observed in adar-/individuals when compared to wild-type at $3 \mathrm{dpf}$ (Fig. 7C). Our findings therefore suggest that the lack of phenotypic alterations in adar-/- larvae, when compared to adar $\mathrm{KD}$ (MO-injected) was not due to genetic compensation. It is possible that the late mortality of zygotic adar KO stems from the presence of maternally deposited adar mRNAs and/or proteins from heterozygous mothers, which might be sufficient to drive early developmental processes in the first few days post fertilization. Altogether, these observations suggest that zygotic Adar is not required for early embryogenesis although its function is still essential for life later on.

\section{Discussion}

\section{Editing primarily in the 3'-UTRs of coding transcripts}

Sequencing data in this work indicate that a very small percentage of A-to-I editing in zebrafish takes place in the protein-coding regions, with the majority occurring in the 3'UTRs of coding transcripts. Editing primarily in the 3'-UTRs and outside the coding regions of transcripts is expected based on similar patterns observed in mammals ${ }^{[51]}$. It is also consistent with a recent data for zebrafish ${ }^{[23]}$ that editing within the proteincoding regions of transcripts sets in after 24 hours. However, we also note that the previous study applied very conservative criteria for editing (to keep the false positive rate low) and called editing sites in embryonic transcripts based on a catalogue of editing sites for adult brain samples ${ }^{[23]}$. Therefore, this study may systematically miss editing sites that are unique for the embryo and not found in the brain. Prior studies have shown that in mammals ADAR2 is more associated with editing in coding sequences, whereas ADAR1 edits more promiscuously, either in transcripts from repetitive genomic elements or, in case of coding transcripts, in the 3'-UTR regions ${ }^{[27]}$. The data in this work confirm that the latter is conserved in zebrafish. Secondary 
structure elements located in 3'-UTR in zebrafish maternal mRNAs have been reported to be involved in translational regulation of mRNAs of the Nodal family by RNA binding proteins ${ }^{[52]}$. Therefore, it is reasonable to expect that RNA editing may participate in such regulation or that similar but distinct secondary structure elements are preferred by Adar in vivo, providing a combinatorial regulatory code for fine-tuning translation and/or degradation rates. The knowledge of secondary structures adopted by maternal mRNAs in vivo is therefore becoming highly necessary to better understand the mechanism of gene expression regulation.

\section{No effect of Adar knockdown on transcript editing prior to the MZT}

The delayed onset of embryonic transcription and reliance on maternal transcript deprives early embryos of the opportunity to regulate gene expression transcriptionally [35]. This limitation, together with the very high adar transcript levels in early zebrafish embryos suggested to us that Adar activity could be used to control transcript and thus protein levels prior to the MZT, as an alternative to transcriptional control. Surprisingly, however, Adar knockdown or overexpression had very limited effect on global A-to-I editing until $5.3 \mathrm{hpf}$, i.e. until well after the MZT at $3.5 \mathrm{hpf}{ }^{[53]}$. Phenotypic data and the editing data for the $12 \mathrm{hpf}$ time point rule out a technical problem with knockout. It is possible (but hard to confirm in the absence of good antibodies) that a sufficient pool of maternal Adar protein is deposited in the oocyte and dominates editing in the early stages (up to $5.3 \mathrm{hpf}$ ), but eventually gets exhausted to reveal editing differences in the knockdown (at 12 hpf). Alternatively, editing changes as a consequence of Adar knockdown could be more subtle than anticipated. For instance, editing pattern alterations could occur within a particular transcript molecule, or different proportions of transcripts for particular genes could be edited as a result of Adar disruption. Unfortunately, the short-read sequencing method used in our study is not able to detect these differences. Long read sequencing methods such as the Oxford Nanopore promise an opportunity to further explore this in the near future.

\section{Different pools of Adar may explain the discrepancy between knockdown and knockout phenotypes}

While the Adar KD by morpholino induced severe patterning defects, adar-/- mutant individuals are viable without obvious embryonic defect. Successful rescue of the knockdown phenotype with a morpholino-resistant transcript makes it unlikely that offtarget effects account for the difference in editing. Genetic compensation was also ruled out in mutants. Thus, the difference in phenotype between Adar KD and mutant could be attributed to distinction of maternal and zygotic function of Adar. Instead, we suspect that maternally deposited Adar transcripts account for the difference. Due to the 
eventual lethality of the adar knockout, the adar-/- embryos were from adar(+/-) heterozygous parents. Therefore, there is a pool of maternally derived adar transcripts in the adar(-/-) embryos. By contrast, translation of the maternally derived adar transcripts is blocked in the morpholino experiments. We suspect that a pool of Adar proteins from embryonic translation of maternal Adar transcript spares the knockout embryo the antero-posterior and dorso-ventral defects that we see in the knockdown embryos. Eventually, however, this Adar pool is probably turned over, so that defects, now purely reflecting the embryonic genotype, become apparent. The detailed causes for this late phenotype are not yet clear. Adar null fish live vastly longer (until the second or third week) than would be expected based on death of the adar1 null mice from hematopoietic defects (at embryonic day 12 in the mouse, equivalent to $36 \mathrm{hpf}$ in zebrafish ${ }^{[54]}$ ). Therefore, similar causes of death are unlikely. Instead, the late phenotype points to other, still unexplored roles of A-to-I editing in late larval development.

\section{Adar mediates embryonic patterning}

Strikingly, knockdown and overexpression of adar resulted in opposite phenotypes, with the former abolishing posterior-ventral structures including the differentiation of notochord, while the latter affecting anterior-dorsal ones resulting in cyclopia. We also found that this phenotype is dependent on an intact RNA editing domain of Adar. These phenotypes are reminiscent of those caused by disruptions to several dorsoventral and anteroposterior axis determinants. Two signaling pathways are known to be responsible for this process: Wnt and FGF. Loss of Wnt signaling is known to cause a severe antero-dorsalized phenotype where embryos possess large heads and truncated tails, while its overactivation results in the opposite phenotype of posteriorized embryos lacking eyes ${ }^{[48,55,56]}$. Similarly, loss of FGF signaling also causes truncation of the posterior body due to the lack of posterior mesoderm structures ${ }^{[57-59]}$. Intriguingly, we observed that several Wnt and FGF pathway components were edited throughout the maternal and zygotic stages, suggesting a link between Adar function and the observed editing events. To our knowledge, a role of A-to-I editing in embryonic patterning is novel and has not been seen before in any vertebrate. Clearly, the very different early embryology of zebrafish compared to mammals creates opportunities for new biological outcomes of A-to-I editing that deserve further study. 


\section{Methods}

\section{Zebrafish}

Zebrafish of wild type (AB strain) and CRISPR/Cas9-generated adar mutant lines were maintained in the zebrafish core facility of the International Institute of Molecular and Cell Biology in Warsaw (IIMCB) (License no. PL14656251) according to standard procedures. Embryos were raised in egg water at $28^{\circ} \mathrm{C}$ and staged according to standard morphological criteria ${ }^{[60]}$.

\section{MO injection, rescue and adar overexpression}

Adar and Adarb1b knockdown was performed using a translation-blocking antisense morpholino oligonucleotides (MO) with the sequences 5'TCCCTCCTCTACCTCTGCTCATAGC-3' and 5'TCCATGATGGTCAAACGTCTCGACT-3' (Gene Tools, USA). For each embryo, 1-3 ng of $\mathrm{MO}$ was injected at the 1-cell stage. For overexpression experiments, the adar cDNA sequence was PCR-amplified using the primer pair 5'-CCTGTCTTTGATACTGTCGTG3' and 5'-TCCCGAAGCCACAGATTCAC-3' and cloned into p-GEMT vector (Promega, USA). For the rescue experiment, wild-type and E1030A mutant adar cDNAs containing a $5 \mathrm{bp}$ mismatch at the $\mathrm{MO}$ recognition site were $\mathrm{PCR}$-amplified using the forward primer

5 CCTAGCTAATACGACTCACTATAGGCGGAACATGAGTAGAGGAAGAGGAGGGAATTAC-3' with a T7 promoter sequence overhang for in vitro transcription and reverse primer 5'TCAAGCTATGCATCCAACGCG-3'. Capped mRNAs for rescue and overexpression were synthesized using the mMessage mMachine T7 Kit (Ambion, USA). Overexpression was done using 25, 50, or $100 \mathrm{pg}$ of mRNA. Rescue experiments were performed using $1 \mathrm{ng}$ of $\mathrm{MO}$ and $25 \mathrm{pg}$ of wild-type or E1030A adar mRNA. Results were obtained from four different experiments on embryos from random pairs.

\section{Disruption of the deaminase domain in adar mRNA}

Zebrafish Adar glutamate 1030 (E1030) in a highly conserved region of the protein (TVNDCHAEIISRRGFIRFLYSELM) was identified as equivalent to human ADAR1 glutamate 912 (E912), a residue known to be indispensable for catalytic activity of the deaminase domain. To encode the E1030A point mutation in the zebrafish adar clone, we used the $Q 5 \AA$ Site-Directed Mutagenesis Kit (NEB) for mutagenic primer-directed replication of both plasmid strands. The first step was an exponential amplification using primers and a master mix formulation of Q5 Hot Start High-Fidelity DNA Polymerase. Oligonucleotides used for glutamate substitution with alanine and site-directed 
mutagenesis are follows: zb_adar_E1030A_F 5'GCAGCTATCATCTCCAGAAGAGGC-3' and zb_adar_E1030A_R 5'ATAGCTGCATGGCAGTCATTTACAG -3'. The second step involved an incubation with an enzyme mix containing a kinase, a ligase and Dpnl allowing for rapid circularization of the PCR product and removal of the template DNA. The last step was a highefficiency transformation into chemically competent TOP10 cells. Selected clones were sent for sequencing and positive ones were linearized and used for in vitro adar E1030A mRNA synthesis.

\section{qPCR for genetic compensation analysis}

Total RNA was extracted from identified (Sanger sequencing) adar KO and wild-type 5 dpf larvae, using TRIZOL LS (Thermo Fisher Scientific, USA) according to the manufacturer's protocol, followed by DNAse I (Life Technologies, USA) treatment. Superscript IV reverse transcriptase (Life Technologies, USA) was used to obtain cDNA. Relative mRNA expression was quantified using FastStart SYBR Green master mix on the LightCycler 96 instrument (Roche Life Science, USA) with specific primer pairs (Supplementary Table 9).

\section{Sequencing and data analysis}

For editing discovery, parental DNA was extracted from one individual male and female from tail-fin clip and sequencing library were synthesized with Nextera XT Kit (Illumina, USA) according to the manufacturer's protocol. RNA from their offspring was also isolated at 16-cell, $3.5 \mathrm{hpf}$, and $5.3 \mathrm{hpf}$ stages. For each time-point, at least 20 embryos were pooled for RNA extraction. To assess the effect of Adar KD and OE, uninjected wild-type and embryos injected with $1 \mathrm{ng}$ of adar MO or $50 \mathrm{pg}$ of adar mRNA were kept until the desired developmental stage: 128-cell, 50\% epiboly, and $12 \mathrm{hpf}$. Two replicates of 20 pooled embryos from the first two time points and three replicates for the $12 \mathrm{hpf}$ time point were isolated for RNA extraction. The RNA for the 12 hpf sample was collected in a second round of experiments, from offspring of a separate mating pair. Total RNA was extracted using TRIzol LS (Thermo Fisher Scientific, USA) and cleaned up on the Qiagen Rneasy Mini column (Qiagen, USA). Quality control of extracted RNA was performed using the 2200 TapeStation system from Agilent Technologies (USA). To avoid the bias caused by cytoplasmic polyadenylation during early embryonic stages, polyA affinity was not used for mRNA enrichment. Instead, total RNA was rRNA-depleted using Ribo-Zero Magnetic Gold Kit (Human, Mouse, Rat; Epicenter). cDNA synthesis for Next-Generation Sequencing (NGS) was performed by SMARTer Stranded RNA-seq kit (Clontech Laboratories, USA) as recommended by the manufacturer. Paired-end sequencing $(2 \times 75$ bp reads) was performed with NextSeq 500 (Illumina, USA). Reads were aligned to the zebrafish genome assembly GRCz10 using STAR v2.7.7a ${ }^{[61]}$ and samtools v1.11 ${ }^{[62]}$. On average, more than $80 \%$ of total 
sequencing reads were uniquely mapped (Supplementary Table 10). Expression quantification was performed using HTSeq v0.11.2 ${ }^{[63]}$. Differential expression was performed using DESeq2 ( $R$ v3.6.3) ${ }^{[64]}$. HTseq reads from KD and OP samples were compared to the control samples at corresponding time points. Multiple testing was done by applying the Benjamini-Hochberg correction as implemented in DESeq2 with adjusted $p$-values $<0.05$ called as statistically significant.

\section{RNA Editing Discovery}

Putative RNA editing sites based on DNA- and RNA-seq input were detected using REDiscover (https://github.com/lpryszcz/REDiscover). The script automatically eliminates low quality and duplicate reads. It utilizes samtools mpileup to generate a text file from input bam files. Options $-q 15-Q 20$, were used, i.e. a minimal mapping quality of 15 and a minimal base call quality of 20 were required. Sites that were not homozygous between female and male samples were excluded from the analysis. Alternative alleles were only called when they were present in at least $20 \%$ of RNA reads. For simplicity, sites with more than one alternative allele were excluded from the analysis. Between 57,605 and 150,495 putative edited sites were detected per sample (Supplementary Table 1). These sites were then filtered in a post-processing step that required a minimum coverage of 10 reads in both the DNA-Seq and RNA-Seq data.

\section{Whole-mount in situ hybridization}

Whole-mount in situ hybridization was performed on 24 hpf wild-type, adar morpholinoinjected and adar mRNA-injected embryos. Dechorionated embryos were fixed in $4 \%$ PFA for 2 hours at room temperature, washed in PBS/Tween (PBT) and then sequentially dehydrated in methanol. After $100 \%$ methanol overnight incubation at $20^{\circ} \mathrm{C}$, the embryos were rehydrated in serial methanol dilutions, washed in PBT, digested with $10 \mu \mathrm{g} / \mathrm{mL}$ Proteinase $\mathrm{K}$ (Roche) for $5 \mathrm{~min}$ and refixed in 4\% PFA for 20 $\mathrm{min}$ at room temperature. Then, they were washed in PBT and incubated in hybridization buffer (Hyb; $5 x$ SSC, $50 \mu \mathrm{g} / \mathrm{ml}$ heparin, $0.1 \%$ Tween $20,500 \mu \mathrm{g} / \mathrm{mL}$ tRNA, $50 \%$ formamide) in a water bath at $68^{\circ} \mathrm{C}$. After 4 hours, the embryos were incubated in hybridization buffer containing $1 \mu \mathrm{g} / \mathrm{mL}$ DIG-labelled RNA probe overnight at $68^{\circ} \mathrm{C}$. Thereafter we performed several post hybridization washes: serial Hyb/2x SSC dilutions for 10 min each at $68^{\circ} \mathrm{C}, 2 \times 10$ min $2 \times S S C$ at $68^{\circ} \mathrm{C}, 2 \times 15 \min 0.2 \times S S C$ at $70^{\circ} \mathrm{C}$ and serial $0.2 x$ SSC/PBT dilutions at room temperature. The embryos were incubated in blocking solution ( $2 \mathrm{mg} / \mathrm{mL}$ BSA, $2 \%$ sheep serum and $1 \times$ PBT) at room temperature for 3-4 hours, after which they were incubated in anti-DIG antibody (Roche, 1:5000) in the dark at $4^{\circ} \mathrm{C}$ overnight. They were washed several times in PBT and incubated in alkaline phosphatase buffer (NTMT; 0.1 M Tris- $\mathrm{HCl} \mathrm{pH} \mathrm{9.5,} 50 \mathrm{mM} \mathrm{MgCl}_{2}, 0.1 \mathrm{M} \mathrm{NaCl}$, 
$0.1 \%$ Tween 20) 3 times for 5 min each. We then stained the embryos by incubating them with NBT/BCIP solution (Roche) at room temperature. When the desired staining intensity was reached, the reaction was stopped by washing the embryos in PBT and fixing them in 4\% PFA. Pictures were taken using Nikon SMZ25 stereomicroscope.

The adar, adarb1a, adarb1b, gsc, foxa2, shhb and tbxta clones were amplified from a cDNA template using specific primers containing a T7 promoter sequence overhang at the reverse primer (Supplementary Table 9), and the corresponding riboprobe was synthesized using the DIG-RNA labeling kit (Roche) according to the manufacturer's instructions. Clones for pax 6 and $t b x 2 b$ were a kind gift from Vladimir Korzh.

\section{CRISPR/Cas9-mediated adar knock-out in zebrafish}

sgRNAs targeting the adar gene were designed using the CCTop on-line tool ${ }^{[65]}$. Three potential sequences were chosen based on their vicinity to the start codon as well as lack of predicted off-target exonic sites. Each sgRNA was tested by co-injecting it with Cas9 mRNA into 1-cell stage embryos. At 24hpf genomic DNA was extracted from single embryos using the HOTSHOT method ${ }^{[66]}$. Genomic DNA was then used as a template for HRM analysis using sets of primers specific to the targeted region of the gene. DNA from single uninjected embryos was used as negative control. Based on the highest percentage of edited embryos, sgRNA_3 (5'- GAGCTGGGGCTTGGGACACG 3') was deemed the most efficient and used for subsequent knock-out line generation. In order to establish the mutant line, embryos of wild-type $(A B)$ line fish at 1-cell stage were injected with 40 pg sgRNA_3 and 400 pg Cas9 mRNA each. The embryos were then raised to adulthood and outcrossed with wild-type AB fish. DNA from embryos resulting from this spawning was extracted and analyzed using the HRM approach as described before to screen for germline transmission ${ }^{[67]}$. Outcross of edit-carrying F0 individuals with wild type resulted in $25 \%$ of heterozygotes (F1). Offspring of incrossed F1 heterozygotes were raised to three months post fertilization and subsequently genotyped to identify mutant homozygotes (adar -/-). Oligonucleotides used for genotyping are as follows: adar_genom_F 5'-CTAACGCTACACCCTCCTCAGC-3' and adar_genom_R 5'- CGTCTGGTACTGGATAGGCTC -3'. Similarly, 3 dpf larvae from F2 generation were genotyped according to a published protocol ${ }^{[68]}$ and sequenced using the abovementioned pair of oligonucleotides.

\section{Data availability}

All sequencing data have been deposited in the GEO database under accession number GSE182714. 


\section{Authors' contributions}

C.W. and M.B. conceived the project and designed the experiments. K. N., C.N.H., E.T., C.W., and M.K. performed experiments. K.A.N. and K.M. prepared libraries and performed sequencing. L.P. developed analyses pipelines and performed data analysis with contribution from M.B.. C.W., M.B., K.N., and E.T. wrote the manuscript. All authors have read and approved the final manuscript.

\section{Acknowledgements}

We are grateful to the zebrafish core facility of the IIMCB Warsaw for excellent fish care; S. Guenther and T. Braun for permission to use sequencing facility and assistance in sequencing runs; M. Łapiński and $M$. Migdał for assistance in data transfer. We thank M. O'Connell, L. Solnica-Krezel, V. Korzh, as well as members of the C.W. and M.B. labs for fruitful discussions.

\section{Funding}

The project no 2015/19/P/NZ2/03655 from National Science Centre, Poland, has received funding from the European Union's Horizon 2020 research and innovation programme under the Marie Skłodowska-Curie grant agreement No 665778, for L.P.. The SONATA Grant no. 2016/21/D/NZ2/03843 from the National Science Center, Poland supports K.N.. The project no. POIR.04.04.00-00-1AF0/16-00* carried out within the First TEAM programme and POIR.04.04.00-00-5D81/17-00** carried out within the TEAM programme of the Foundation for Polish Science co-financed by the European Union under the European Regional Development Fund support C.W.*, K.A.N.*, and M.B**.. The OPUS grant no. 2019/35/B/NZ2/02548 supports C.W.. The project no 2018/30/Q/NZ2/00669 from National Science Centre, Poland, and project no PPI/APM/2018/1/00034 from the Polish National Agency for Academic Exchange supports M.B.. This work was supported by EU/FP7 - Research Potential FISHMED, grant number: 316125 . 


\section{References}

1. Gott, J. M., \& Emeson, R. B. (2000). Functions and mechanisms of RNA editing. Annual Review of Genetics, 34, 499-531. https://doi.org/10.1146/annurev.genet.34.1.499

2. Zinshteyn, B., \& Nishikura, K. (2009). Adenosine-to-inosine RNA editing. WIREs Systems Biology and Medicine, 1(2), 202-209. https://doi.org/10.1002/wsbm.10

3. Nishikura, K. (2010). Functions and Regulation of RNA Editing by ADAR Deaminases. Annual Review of Biochemistry, 79, 321-349. https://doi.org/10.1146/annurev-biochem060208-105251

4. Wahlstedt, H., Daniel, C., Ensterö, M., \& Öhman, M. (2009). Large-scale mRNA sequencing determines global regulation of RNA editing during brain development. Genome Research, 19(6), 978-986. https://doi.org/10.1101/gr.089409.108

5. Higuchi, M., Maas, S., Single, F. N., Hartner, J., Rozov, A., Burnashev, N., Feldmeyer, D., Sprengel, R., \& Seeburg, P. H. (2000). Point mutation in an AMPA receptor gene rescues lethality in mice deficient in the RNA-editing enzyme ADAR2. Nature, 406(6791), 78-81. https://doi.org/10.1038/35017558

6. Porath, H. T., Schaffer, A. A., Kaniewska, P., Alon, S., Eisenberg, E., Rosenthal, J., Levanon, E. Y., \& Levy, O. (2017). A-to-I RNA Editing in the Earliest-Diverging Eumetazoan Phyla. Molecular Biology and Evolution, 34(8), 1890-1901. https://doi.org/10.1093/molbev/msx125

7. Yablonovitch, A. L., Deng, P., Jacobson, D., \& Li, J. B. (2017). The evolution and adaptation of A-to-I RNA editing. PLoS Genetics, 13(11), e1007064. https://doi.org/10.1371/journal.pgen.1007064

8. Liddicoat, B. J., Piskol, R., Chalk, A. M., Ramaswami, G., Higuchi, M., Hartner, J. C., Li, J. B., Seeburg, P. H., \& Walkley, C. R. (2015). RNA editing by ADAR1 prevents MDA5 sensing of endogenous dsRNA as nonself. Science (New York, N.Y.), 349(6252), 11151120. https://doi.org/10.1126/science.aac7049

9. Deng, P., Khan, A., Jacobson, D., Sambrani, N., McGurk, L., Li, X., Jayasree, A., Hejatko, J., Shohat-Ophir, G., O'Connell, M. A., Li, J. B., \& Keegan, L. P. (2020). Adar RNA editingdependent and -independent effects are required for brain and innate immune functions in Drosophila. Nature Communications, 11(1), 1580. https://doi.org/10.1038/s41467-02015435-1

10. Quin, J., Sedmík, J., Vukić, D., Khan, A., Keegan, L. P., \& O’Connell, M. A. (2021). ADAR RNA Modifications, the Epitranscriptome and Innate Immunity. Trends in Biochemical Sciences, 46(9), 758-771. https://doi.org/10.1016/j.tibs.2021.02.002

11. Lamers, M. M., van den Hoogen, B. G., \& Haagmans, B. L. (2019). ADAR1: "Editor-in-Chief" of Cytoplasmic Innate Immunity. Frontiers in Immunology, 10, 1763. https://doi.org/10.3389/fimmu.2019.01763

12. Eisenberg, E., \& Levanon, E. Y. (2018). A-to-I RNA editing—Immune protector and transcriptome diversifier. Nature Reviews Genetics, 19(8), 473-490. https://doi.org/10.1038/s41576-018-0006-1

13. Higuchi, M., Single, F. N., Köhler, M., Sommer, B., Sprengel, R., \& Seeburg, P. H. (1993). RNA editing of AMPA receptor subunit GluR-B: A base-paired intron-exon structure 
determines position and efficiency. Cell, 75(7), 1361-1370. https://doi.org/10.1016/00928674(93)90622-w

14. Li, I.-C., Chen, Y.-C., Wang, Y.-Y., Tzeng, B.-W., Ou, C.-W., Lau, Y.-Y., Wu, K.-M., Chan, T.-M., Lin, W.-H., Hwang, S.-P. L., \& Chow, W.-Y. (2014). Zebrafish Adar2 Edits the Q/R Site of AMPA Receptor Subunit gria2a Transcript to Ensure Normal Development of Nervous System and Cranial Neural Crest Cells. PLOS ONE, 9(5). https://doi.org/10.1371/journal.pone.0097133

15. Stapleton, M., Carlson, J. W., \& Celniker, S. E. (2006). RNA editing in Drosophila melanogaster: New targets and functional consequences. RNA, 12(11), 1922-1932. https://doi.org/10.1261/rna.254306

16. Li, Q., Wang, Z., Lian, J., Schiøtt, M., Jin, L., Zhang, P., Zhang, Y., Nygaard, S., Peng, Z., Zhou, Y., Deng, Y., Zhang, W., Boomsma, J. J., \& Zhang, G. (2014). Caste-specific RNA editomes in the leaf-cutting ant Acromyrmex echinatior. Nature Communications, 5(1), 4943. https://doi.org/10.1038/ncomms5943

17. Alon, S., Garrett, S. C., Levanon, E. Y., Olson, S., Graveley, B. R., Rosenthal, J. J. C., \& Eisenberg, E. (2015). The majority of transcripts in the squid nervous system are extensively recoded by A-to-I RNA editing. ELife, 4, e05198. https://doi.org/10.7554/eLife.05198

18. Vallecillo-Viejo, I. C., Liscovitch-Brauer, N., Diaz Quiroz, J. F., Montiel-Gonzalez, M. F., Nemes, S. E., Rangan, K. J., Levinson, S. R., Eisenberg, E., \& Rosenthal, J. J. C. (2020). Spatially regulated editing of genetic information within a neuron. Nucleic Acids Research, 48(8), 3999-4012. https://doi.org/10.1093/nar/gkaa172

19. Levanon, E. Y., \& Eisenberg, E. (2015). Does RNA editing compensate for Alu invasion of the primate genome? BioEssays, 37(2), 175-181. https://doi.org/10.1002/bies.201400163

20. Athanasiadis, A., Rich, A., \& Maas, S. (2004). Widespread A-to-I RNA Editing of AluContaining mRNAs in the Human Transcriptome. PLOS Biology, 2(12), e391. https://doi.org/10.1371/journal.pbio.0020391

21. Kim, D. D. Y., Kim, T. T. Y., Walsh, T., Kobayashi, Y., Matise, T. C., Buyske, S., \& Gabriel, A. (2004). Widespread RNA editing of embedded alu elements in the human transcriptome. Genome Research, 14(9), 1719-1725. https://doi.org/10.1101/gr.2855504

22. Neeman, Y., Levanon, E. Y., Jantsch, M. F., \& Eisenberg, E. (2006). RNA editing level in the mouse is determined by the genomic repeat repertoire. $R N A, 12(10), 1802-1809$. https://doi.org/10.1261/rna.165106

23. Buchumenski, I., Holler, K., Appelbaum, L., Eisenberg, E., Junker, J. P., \& Levanon, E. Y. (2021). Systematic identification of A-to-I RNA editing in zebrafish development and adult organs. Nucleic Acids Research, 49(8), 4325-4337. https://doi.org/10.1093/nar/gkab247

24. Keegan, L. P., McGurk, L., Palavicini, J. P., Brindle, J., Paro, S., Li, X., Rosenthal, J. J. C., \& O'Connell, M. A. (2011). Functional conservation in human and Drosophila of Metazoan ADAR2 involved in RNA editing: Loss of ADAR1 in insects. Nucleic Acids Research, 39(16), 7249-7262. https://doi.org/10.1093/nar/gkr423 
25. Savva, Y. A., Rieder, L. E., \& Reenan, R. A. (2012). The ADAR protein family. Genome Biology, 13(12), 252. https://doi.org/10.1186/gb-2012-13-12-252

26. Chen, C. X., Cho, D. S., Wang, Q., Lai, F., Carter, K. C., \& Nishikura, K. (2000). A third member of the RNA-specific adenosine deaminase gene family, ADAR3, contains both single- and double-stranded RNA binding domains. RNA, 6(5), 755-767.

27. Costa Cruz, P. H., Kato, Y., Nakahama, T., Shibuya, T., \& Kawahara, Y. (2020). A comparative analysis of ADAR mutant mice reveals site-specific regulation of RNA editing. RNA (New York, N.Y.), 26(4), 454-469. https://doi.org/10.1261/rna.072728.119

28. Chen, C. X., Cho, D. S., Wang, Q., Lai, F., Carter, K. C., \& Nishikura, K. (2000). A third member of the RNA-specific adenosine deaminase gene family, ADAR3, contains both single- and double-stranded RNA binding domains. RNA, 6(5), 755-767.

29. Sun, T., Yu, Y., Wu, X., Acevedo, A., Luo, J.-D., Wang, J., Schneider, W. M., Hurwitz, B., Rosenberg, B. R., Chung, H., \& Rice, C. M. (2021). Decoupling expression and editing preferences of ADAR1 p150 and p110 isoforms. Proceedings of the National Academy of Sciences of the United States of America, 118(12), e2021757118. https://doi.org/10.1073/pnas.2021757118

30. Patterson, J. B., \& Samuel, C. E. (1995). Expression and regulation by interferon of a double-stranded-RNA-specific adenosine deaminase from human cells: Evidence for two forms of the deaminase. Molecular and Cellular Biology, 15(10), 5376-5388.

31. Wang, Q., Khillan, J., Gadue, P., \& Nishikura, K. (2000). Requirement of the RNA editing deaminase ADAR1 gene for embryonic erythropoiesis. Science (New York, N.Y.), 290(5497), 1765-1768. https://doi.org/10.1126/science.290.5497.1765

32. Hartner, J. C., Schmittwolf, C., Kispert, A., Müller, A. M., Higuchi, M., \& Seeburg, P. H. (2004). Liver disintegration in the mouse embryo caused by deficiency in the RNAediting enzyme ADAR1. The Journal of Biological Chemistry, 279(6), 4894-4902. https://doi.org/10.1074/jbc.M311347200

33. Wang, Q., Miyakoda, M., Yang, W., Khillan, J., Stachura, D. L., Weiss, M. J., \& Nishikura, K. (2004). Stress-induced apoptosis associated with null mutation of ADAR1 RNA editing deaminase gene. The Journal of Biological Chemistry, 279(6), 4952-4961. https://doi.org/10.1074/jbc.M310162200

34. Gallo, A., Vukic, D., Michalík, D., O'Connell, M. A., \& Keegan, L. P. (2017). ADAR RNA editing in human disease; more to it than meets the I. Human Genetics, 136(9), 12651278. https://doi.org/10.1007/s00439-017-1837-0

35. Winata, C. L., Nama, S., Hajan, H. S., Lee, S. G. P., Korzh, V., Sampath, P., Tanavde, V., \& Mathavan, S. (2018). Cytoplasmic polyadenylation-mediated translational control of maternal mRNAs directs maternal-to-zygotic transition. 12.

36. Heale, B. S. E., Keegan, L. P., McGurk, L., Michlewski, G., Brindle, J., Stanton, C. M., Caceres, J. F., \& O'Connell, M. A. (2009). Editing independent effects of ADARs on the miRNA/siRNA pathways. The EMBO Journal, 28(20), 3145-3156.

https://doi.org/10.1038/emboj.2009.244

37. Krauss, S., Johansen, T., Korzh, V., \& Fjose, A. (1991). Expression pattern of zebrafish pax genes suggests a role in early brain regionalization. Nature, 353(6341), 267-270. https://doi.org/10.1038/353267a0 
38. Dheen, T., Sleptsova-Friedrich, I., Xu, Y., Clark, M., Lehrach, H., Gong, Z., \& Korzh, V. (1999). Zebrafish tbx-c functions during formation of midline structures. Development (Cambridge, England), 126(12), 2703-2713.

39. Chuang, J. C., \& Raymond, P. A. (2002). Embryonic origin of the eyes in teleost fish. BioEssays: News and Reviews in Molecular, Cellular and Developmental Biology, 24(6), 519-529. https://doi.org/10.1002/bies.10097

40. Heisenberg, C. P., Tada, M., Rauch, G. J., Saúde, L., Concha, M. L., Geisler, R., Stemple, D. L., Smith, J. C., \& Wilson, S. W. (2000). Silberblick/Wnt11 mediates convergent extension movements during zebrafish gastrulation. Nature, 405(6782), 76-81. https://doi.org/10.1038/35011068

41. Myers, D. C., Sepich, D. S., \& Solnica-Krezel, L. (2002). Bmp activity gradient regulates convergent extension during zebrafish gastrulation. Developmental Biology, 243(1), 8198. https://doi.org/10.1006/dbio.2001.0523

42. Simonis, N., Migeotte, I., Lambert, N., Perazzolo, C., de Silva, D. C., Dimitrov, B., Heinrichs, C., Janssens, S., Kerr, B., Mortier, G., Van Vliet, G., Lepage, P., Casimir, G., Abramowicz, M., Smits, G., \& Vilain, C. (2013). FGFR1 mutations cause Hartsfield syndrome, the unique association of holoprosencephaly and ectrodactyly. Journal of Medical Genetics, 50(9), 585-592. https://doi.org/10.1136/jmedgenet-2013-101603

43. Walker, M. B., Miller, C. T., Coffin Talbot, J., Stock, D. W., \& Kimmel, C. B. (2006). Zebrafish furin mutants reveal intricacies in regulating Endothelin1 signaling in craniofacial patterning. Developmental Biology, 295(1), 194-205. https://doi.org/10.1016/j.ydbio.2006.03.028

44. Brown, J. L., Snir, M., Noushmehr, H., Kirby, M., Hong, S.-K., Elkahloun, A. G., \& Feldman, B. (2008). Transcriptional profiling of endogenous germ layer precursor cells identifies dusp4 as an essential gene in zebrafish endoderm specification. Proceedings of the National Academy of Sciences of the United States of America, 105(34), 12337-12342. https://doi.org/10.1073/pnas.0805589105

45. Schepis, A., Sepich, D., \& Nelson, W. J. (2012). AE-catenin regulates cell-cell adhesion and membrane blebbing during zebrafish epiboly. Development (Cambridge, England), 139(3), 537-546. https://doi.org/10.1242/dev.073932

46. Davidson, A. J., Ernst, P., Wang, Y., Dekens, M. P. S., Kingsley, P. D., Palis, J., Korsmeyer, S. J., Daley, G. Q., \& Zon, L. I. (2003). Cdx4 mutants fail to specify blood progenitors and can be rescued by multiple hox genes. Nature, 425(6955), 300-306. https://doi.org/10.1038/nature01973

47. Martyn, U., \& Schulte-Merker, S. (2003). The ventralized ogon mutant phenotype is caused by a mutation in the zebrafish homologue of Sizzled, a secreted Frizzled-related protein. Developmental Biology, 260(1), 58-67. https://doi.org/10.1016/s0012-1606(03)00221-5

48. Shimizu, T., Bae, Y.-K., Muraoka, O., \& Hibi, M. (2005). Interaction of Wnt and caudalrelated genes in zebrafish posterior body formation. Developmental Biology, 279(1), 125-141. https://doi.org/10.1016/j.ydbio.2004.12.007

49. Prince, V. E., Joly, L., Ekker, M., \& Ho, R. K. (1998). Zebrafish hox genes: Genomic organization and modified colinear expression patterns in the trunk. Development (Cambridge, England), 125(3), 407-420. 
50. El-Brolosy, M. A., Kontarakis, Z., Rossi, A., Kuenne, C., Günther, S., Fukuda, N., Kikhi, K., Boezio, G. L. M., Takacs, C. M., Lai, S.-L., Fukuda, R., Gerri, C., Giraldez, A. J., \& Stainier, D. Y. R. (2019). Genetic compensation triggered by mutant mRNA degradation. Nature, 568(7751), 193-197. https://doi.org/10.1038/s41586-019-1064-z

51. DARNED: a DAtabase of RNa EDiting in humans—PubMed. (n.d.). Retrieved August 16, 2021, from https://pubmed.ncbi.nlm.nih.gov/20547637/

52. Zaucker, A., Nagorska, A., Kumari, P., Hecker, N., Wang, Y., Huang, S., Cooper, L., Sivashanmugam, L., VijayKumar, S., Brosens, J., Gorodkin, J., \& Sampath, K. (2018). Translational co-regulation of a ligand and inhibitor by a conserved RNA element. Nucleic Acids Research, 46(1), 104-119. https://doi.org/10.1093/nar/gkx938

53. Aanes, H., Winata, C. L., Lin, C. H., Chen, J. P., Srinivasan, K. G., Lee, S. G. P., Lim, A. Y. M., Hajan, H. S., Collas, P., Bourque, G., Gong, Z., Korzh, V., Aleström, P., \& Mathavan, S. (2011). Zebrafish mRNA sequencing deciphers novelties in transcriptome dynamics during maternal to zygotic transition. Genome Research, 21(8), 1328-1338. https://doi.org/10.1101/gr.116012.110

54. Kulkeaw, K., \& Sugiyama, D. (2012). Zebrafish erythropoiesis and the utility of fish as models of anemia. Stem Cell Research \& Therapy, 3(6), 55. https://doi.org/10.1186/scrt146

55. Chen, Q., Takada, R., \& Takada, S. (2012). Loss of Porcupine impairs convergent extension during gastrulation in zebrafish. Journal of Cell Science, 125(Pt 9), 2224-2234. https://doi.org/10.1242/jcs.098368

56. Hino, H., Nakanishi, A., Seki, R., Aoki, T., Yamaha, E., Kawahara, A., Shimizu, T., \& Hibi, M. (2018). Roles of maternal wnt8a transcripts in axis formation in zebrafish.

Developmental Biology, 434(1), 96-107. https://doi.org/10.1016/j.ydbio.2017.11.016

57. Draper, B. W., Stock, D. W., \& Kimmel, C. B. (2003). Zebrafish fgf24 functions with fgf8 to promote posterior mesodermal development. Development (Cambridge, England), 130(19), 4639-4654. https://doi.org/10.1242/dev.00671

58. Fürthauer, M., Van Celst, J., Thisse, C., \& Thisse, B. (2004). Fgf signalling controls the dorsoventral patterning of the zebrafish embryo. Development (Cambridge, England), 131(12), 2853-2864. https://doi.org/10.1242/dev.01156

59. Maegawa, S., Varga, M., \& Weinberg, E. S. (2006). FGF signaling is required for \{beta\}catenin-mediated induction of the zebrafish organizer. Development (Cambridge, England), 133(16), 3265-3276. https://doi.org/10.1242/dev.02483

60. Kimmel, C. B., Ballard, W. W., Kimmel, S. R., Ullmann, B., \& Schilling, T. F. (1995). Stages of embryonic development of the zebrafish. Developmental Dynamics: An Official Publication of the American Association of Anatomists, 203(3), 253-310. https://doi.org/10.1002/aja.1002030302

61. Dobin, A., Davis, C. A., Schlesinger, F., Drenkow, J., Zaleski, C., Jha, S., Batut, P., Chaisson, M., \& Gingeras, T. R. (2013). STAR: Ultrafast universal RNA-seq aligner. Bioinformatics (Oxford, England), 29(1), 15-21. https://doi.org/10.1093/bioinformatics/bts635

62. Li, H., Handsaker, B., Wysoker, A., Fennell, T., Ruan, J., Homer, N., Marth, G., Abecasis, G., Durbin, R., \& 1000 Genome Project Data Processing Subgroup. (2009). The 
Sequence Alignment/Map format and SAMtools. Bioinformatics (Oxford, England), 25(16), 2078-2079. https://doi.org/10.1093/bioinformatics/btp352

63. Anders, S., Pyl, P. T., \& Huber, W. (2015). HTSeq-A Python framework to work with highthroughput sequencing data. Bioinformatics, 31(2), 166-169.

https://doi.org/10.1093/bioinformatics/btu638

64. Love, M. I., Huber, W., \& Anders, S. (2014). Moderated estimation of fold change and dispersion for RNA-seq data with DESeq2. Genome Biology, 15(12), 550. https://doi.org/10.1186/s13059-014-0550-8

65. Stemmer, M., Thumberger, T., Keyer, M. del S., Wittbrodt, J., \& Mateo, J. L. (2015). CCTop: An Intuitive, Flexible and Reliable CRISPR/Cas9 Target Prediction Tool. PLOS ONE, 10(4), e0124633. https://doi.org/10.1371/journal.pone.0124633

66. Meeker, N. D., Hutchinson, S. A., Ho, L., \& Trede, N. S. (2007). Method for isolation of PCRready genomic DNA from zebrafish tissues. BioTechniques, 43(5), 610, 612, 614. https://doi.org/10.2144/000112619

67. Parant, J. M., George, S. A., Pryor, R., Wittwer, C. T., \& Yost, H. J. (2009). A Rapid and Efficient Method of Genotyping Zebrafish Mutants. Developmental Dynamics : An Official Publication of the American Association of Anatomists, 238(12), 3168-3174. https://doi.org/10.1002/dvdy.22143

68. Kosuta, C., Daniel, K., Johnstone, D. L., Mongeon, K., Ban, K., LeBlanc, S., MacLeod, S., Et-Tahiry, K., Ekker, M., MacKenzie, A., \& Pena, I. (2018). High-throughput DNA Extraction and Genotyping of 3dpf Zebrafish Larvae by Fin Clipping. Journal of Visualized Experiments: JoVE, 136. https://doi.org/10.3791/58024 


\section{Figure Legends}

Figure 1. Spatiotemporal expression pattern of Adar family genes in zebrafish. (A) Expression of zebrafish adar family based on transcriptome profiling of developing embryos (RNA-seq) ${ }^{[54]}$. Expression levels are plotted for developmental stages: egg, 1 cell, 16 cells, 128 cells, $3.5 \mathrm{hpf}$ and $5.3 \mathrm{hpf}$. Levels of $(A)$ total RNA and (B) polysomeassociated RNA of four zebrafish adar paralogs are given. Whole mount in situ hybridization shows expression pattern of $\operatorname{adar}(\mathrm{C}, \mathrm{D})$ and $\operatorname{adarb} 1 b(\mathrm{E}, \mathrm{F})$ in $24 \mathrm{hpf}$ zebrafish embryos.

Figure 2. Phenotypic defects at $24 \mathrm{hpf}$ caused by adar knockdown and overexpression. (A) Wild-type (B, C) Adar MO-injected embryos develop abnormal phenotype in the posterior part with disturbed body axis, shortened tail and crooked, disorganized notochord. (D) MO phenotype can be fully rescued with wild-type mRNA injection. (E) Mutant adar mRNA E1030A with inactivated editing domain could not rescue the malformed phenotype. (F,G) Phenotype defects caused by adar mRNA overexpression. The anterior defects, including cyclopia and head malformations are mRNA dose-dependent. Inset marked by red boxes denotes overlaid identical image taken at different focal plane. $(\mathrm{H}, \mathrm{I})$ Injection statistics of Adar $\mathrm{MO}$, rescue, and mRNA overexpression.

Figure 3. Embryonic patterning defects due to Adar loss- or gain-of-function. Whole mount in situ hybridization detection of marker genes in Adar morpholino knockdown and adar mRNA overexpression. (A-F) pax6 expression demarcates anterior brain structures including optic vesicle (OpV), telencephalon (TE), and diencephalon (DE) as well as hindbrain and anterior spinal cord. (G-L) tbx2b expression marking TE, dorsal part of the OpV, DE, and otic vesicle (OV). (M-O) expression of tbxta marks the notochord. (P, Q) shhb expression indicates floor plate (FP) and midbrain-hindbrain boundary (MHB). 
Figure 4. A-to-I RNA editing sites in early embryonic development. (A) Schematics of A-to-I RNA editing discovery through sequencing of a parent-offspring trio. The genome sequence of the parents are used as a reference set to distinguish between polymorphisms and editing. (B) Mismatches between RNA and DNA sequencing data. As RNA libraries were not strand selective, mismatches were read as their complement (i.e. T->C instead of $A->G$, or $C->T$ as $G->A$ ) in roughly half of all cases. (C) Overlap of editing sites at different time points. The 1.5 and $3.5 \mathrm{hpf}$ samples were more similar to each other than to the $5.3 \mathrm{hpf}$ sample, probably because of replacement of maternal by zygotic transcripts at the MZT. (D) Association of editing sites with genomic features. A large fraction of RNA editing is classified as 'genic_other' due to overlap between introns/exons/UTRs from multiple transcripts. (E) Number of editing sites in transcripts stemming from different classes of repeat elements. (F) Number of editing events in individual reads encompassing 10 potential editing sites. The majority of individual reads contained 1-3 RNA editing sites, and never more than 5 editing sites.

Figure 5. Selected transcripts of developmental signaling pathway genes implicated in dorsoventral and/or anteroposterior patterning containing two or more RNA editing sites detected at their 3'-UTR region. Gene names in red denotes those that are commonly edited at all three stages.

Figure 6. Differentially expressed genes at $12 \mathrm{hpf}$ in Adar KD and OE: (A) Principal component analysis of control, Adar knockdown and overexpression samples based on their transcriptome profile. (B) Number of genes differentially expressed in each condition and their overlap. (C) Differential expression analysis of Adar KD and OP compared to control. Genes with significant change in expression $(p<0.05)$ in red.

Figure 7. Generation of adar-/- mutant by CRISPR/Cas9. (A) adar-/- mutant had a 5bp deletion within the second exon, resulting in a premature stop codon at $115 \mathrm{bp}$ position. (B) Different genotype ratios at different time-points suggesting that adar-/homozygotes die between $3 \mathrm{dpf}$ and adult stage. (C) qRT-PCR of adar and its paralogs shows the lack of genetic compensation effect in homozygous mutant individuals. 


\section{Suppl. Figure Legends}

Supplementary Figure 1. Adar and Adarb1b knockdown experiments. (A) Dosedependent effect of Adar MO was observed starting from $5.3 \mathrm{hpf}$, while Adarb1b MO did not cause any observable phenotype. (B) Design of adar mutant mRNA E1030A with point mutation abolishing the activity of the deaminase domain.

\section{Supplementary Figure 2. Translation rates and number of editing sites for $\mathbf{1 . 5} \mathrm{hpf}$} and $5.3 \mathrm{hpf}$ transcripts. Translation rates are expressed as ratio of polysome bound to sum of bound and unbound fractions of a given transcript ${ }^{[54]}$. Thus, translation rate of 1.0 means all expressed transcript molecules are associated with polysome, while 0.0 means none of expressed transcript molecules are associated with polysome.

Supplementary Figure 3. Comparison of expression levels between two developmental stages of non-edited, low-edited, and highly edited transcripts.

Supplementary Figure 4. Global RNA editing and gene expression profile of Adar knockdown and overexpression. (A) Penetrance of RNA editing expressed as number of transcripts vs. fraction of editing within a transcript species in 128-cell (left column) and $5.3 \mathrm{hpf}$ (right column). (B) Principal component analysis of control, Adar knockdown and overexpression samples based on their transcriptome profile. (C) Differential expression analysis of Adar knockdown and overexpression vs. control. No genes were differentially expressed at $p<0.05$.

Supplementary Figure 5. Comparison of RNA editing frequency between control and Adar KD or OE. RNA editing frequencies for each transcript is plotted for each sample in 128-cell and 12 hpf stages. Spearman's rank correlation coefficient $(\rho)$ is given in every figure title. 

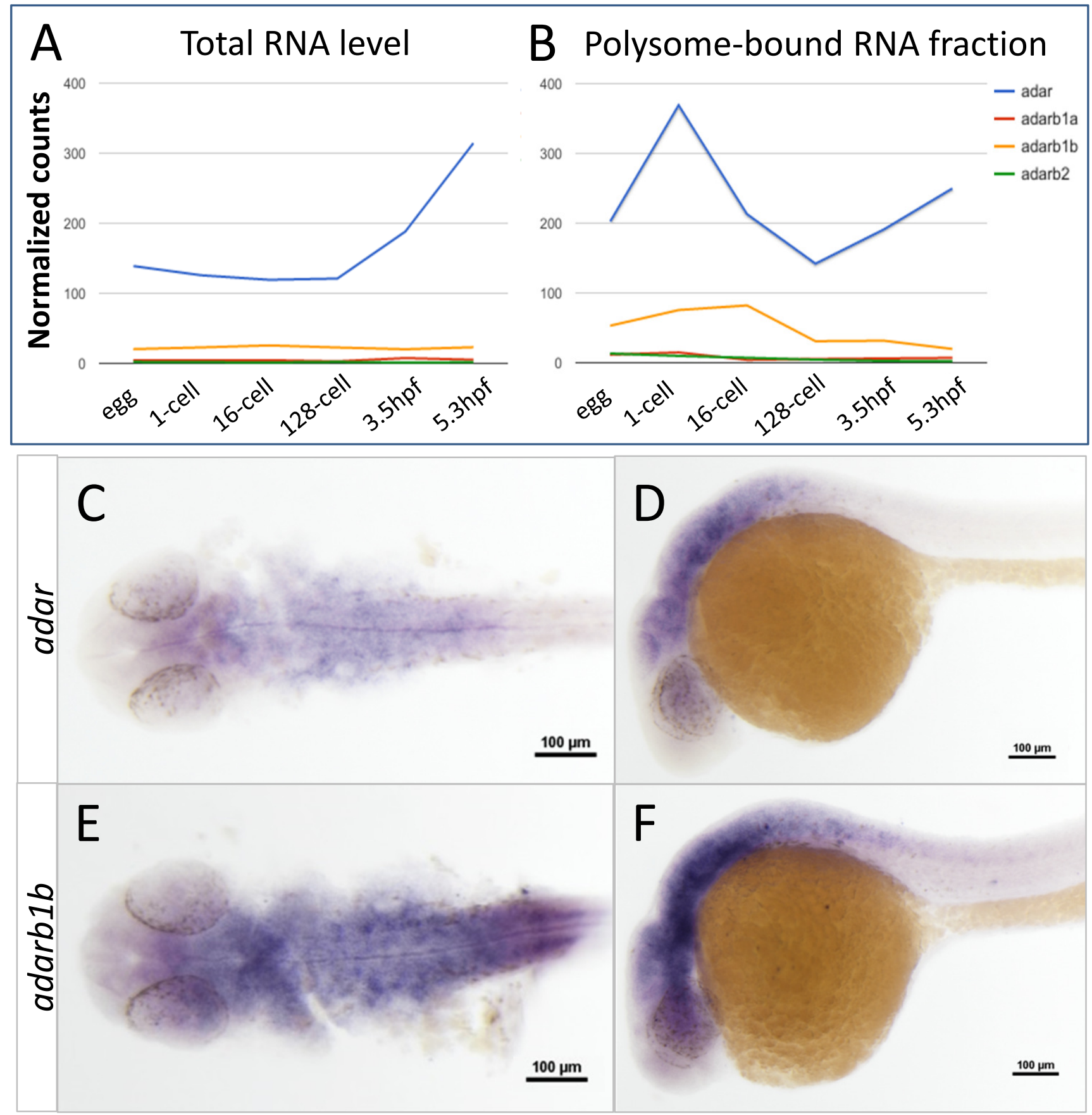

Figure 1 


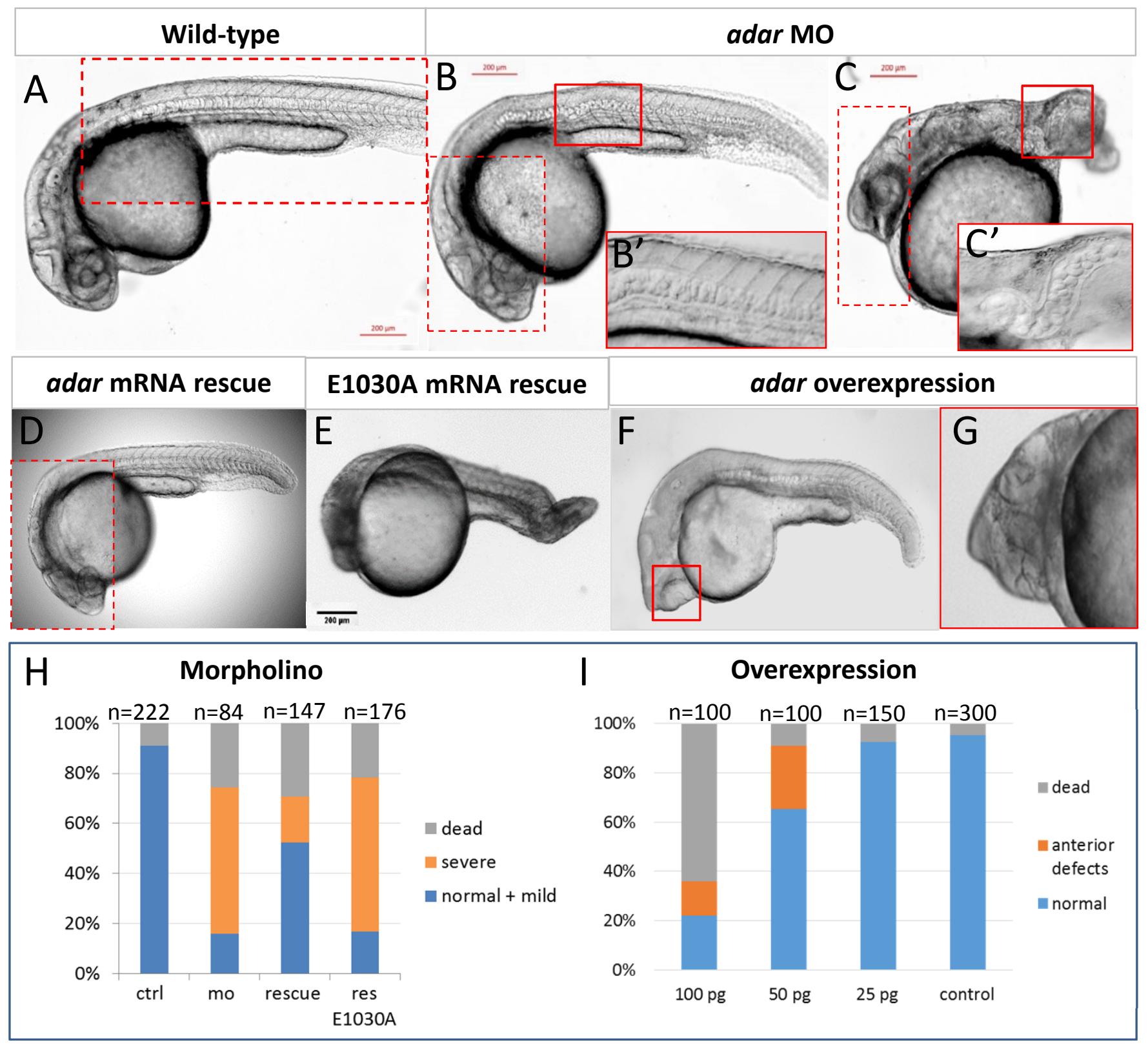

Figure 2 


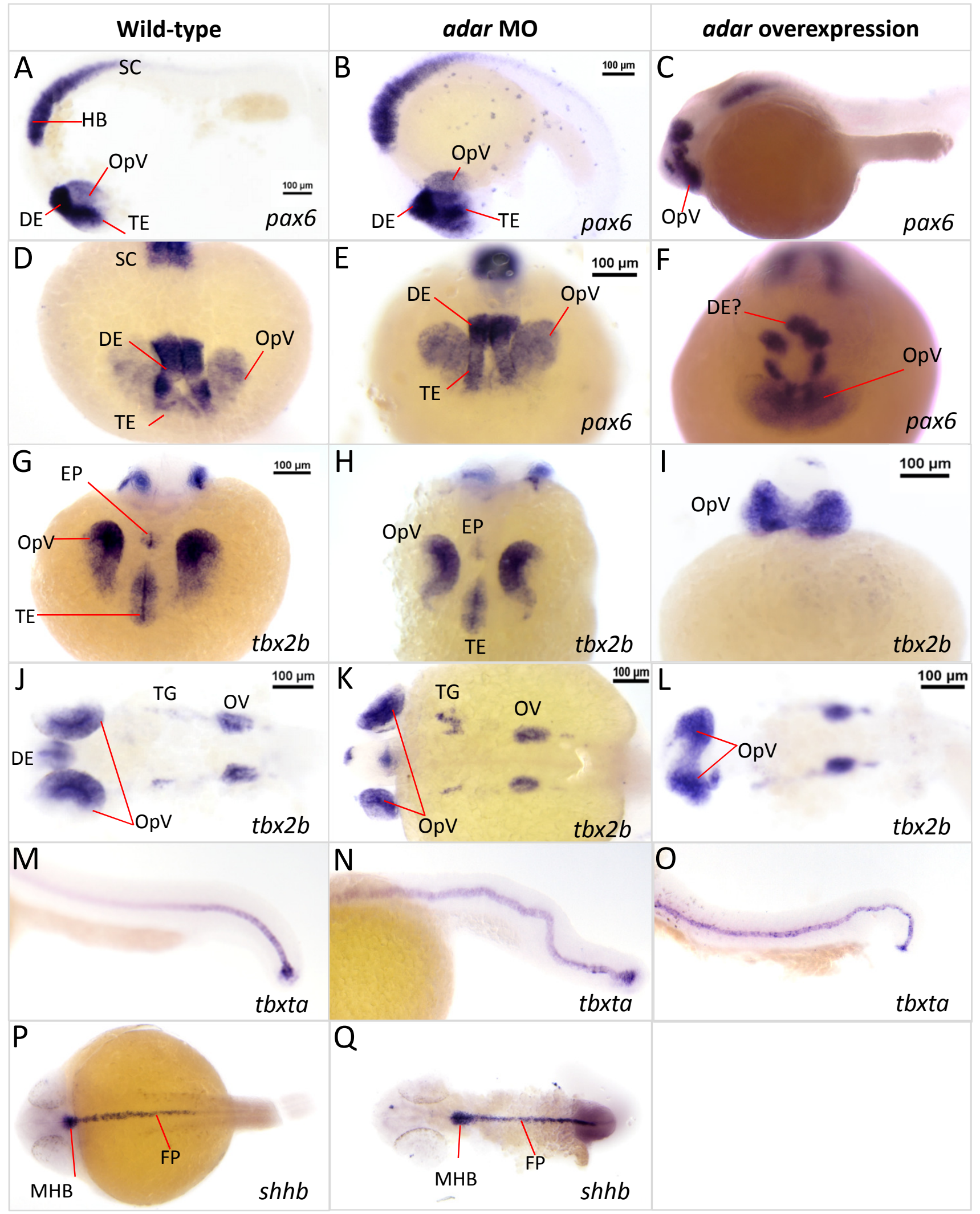

Figure 3 


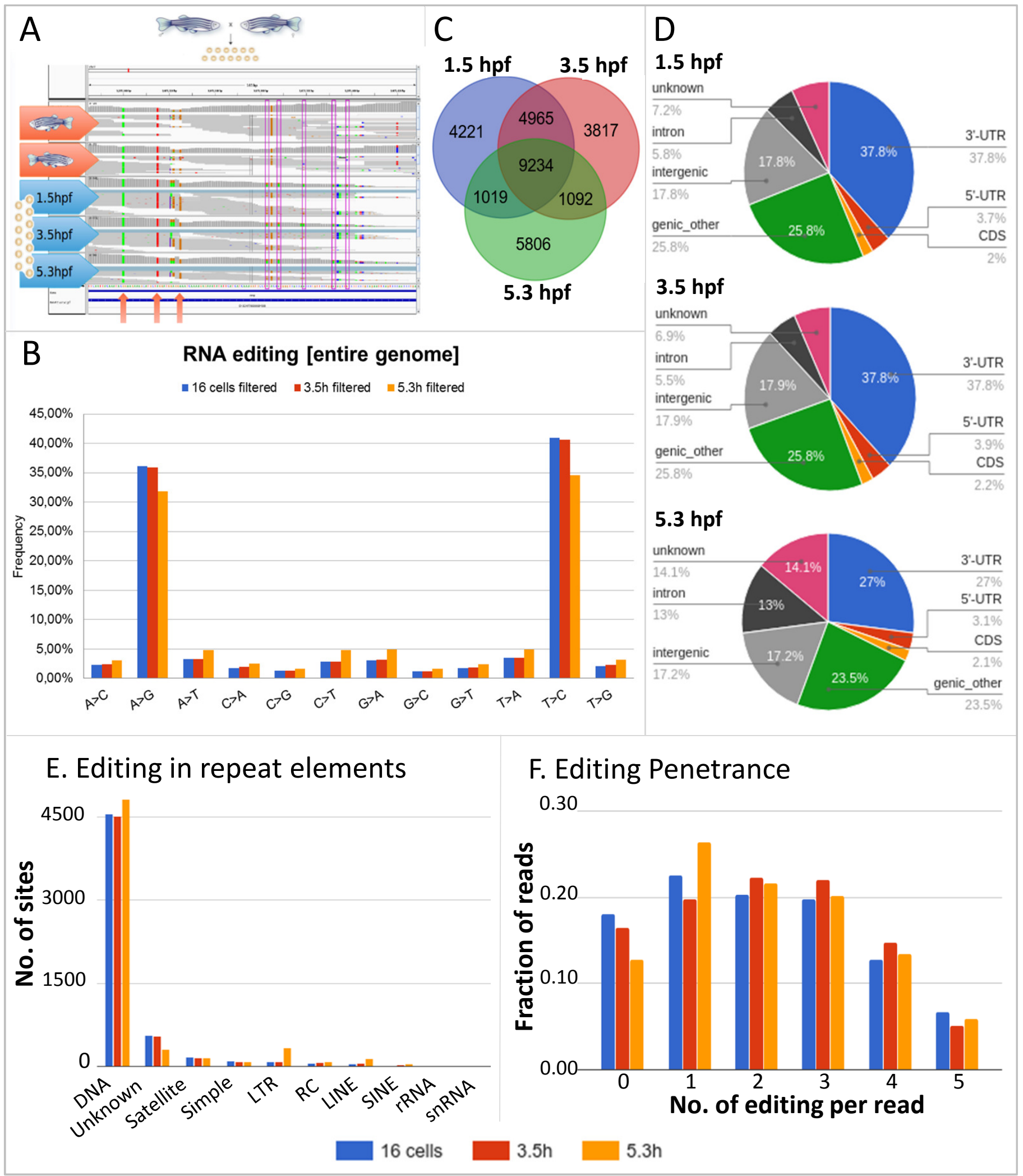

Figure 4 


\begin{tabular}{|c|c|c|c|c|c|}
\hline \multicolumn{2}{|c|}{$1.5 \mathrm{hpf}$} & \multicolumn{2}{|c|}{$3.5 \mathrm{hpf}$} & \multicolumn{2}{|c|}{$5.3 \mathrm{hpf}$} \\
\hline $\begin{array}{l}\text { Cell migration } \\
\text { jak1 } \\
\text { abl2 } \\
\text { fermt2 } \\
\text { nr6a1a } \\
\text { ndel1b } \\
\text { usp9 } \\
\text { Neurogenesis } \\
\text { cdk10 } \\
\text { Ilgl1 } \\
\text { nr6a1a } \\
\text { pafah1b1a/b } \\
\text { Chordate } \\
\text { embryonic dvp } \\
\text { adrbk2 } \\
\text { igf1rb } \\
\text { ambra1b } \\
\text { hip1 } \\
\text { insrb } \\
\text { ocrl } \\
\text { rnf170 } \\
\text { tp53bp2a }\end{array}$ & $\begin{array}{l}\text { Wnt signaling } \\
d v / 2 \\
f e r m t 2 \\
f z d 3 b \\
f z d 5 \\
f z d 8 b \\
\text { metap } 2 b \\
\text { Rnf146 } \\
\text { tcf7l1b } \\
\text { FGF signaling } \\
\text { fgfr1a } \\
\text { ext/3 } \\
\text { Epiboly } \\
\text { ezrb } \\
\text { Endoderm } \\
\text { dusp4 } \\
\text { Craniofacial dvp } \\
\text { furina }\end{array}$ & $\begin{array}{l}\text { Neurogenesis } \\
\text { cdk10 } \\
\text { Ilgl1 } \\
\text { nr6a1a } \\
\text { pafah1b1a/b } \\
\text { Wnt signaling } \\
d v / 2 \\
\text { fermt2 } \\
\text { fzd3b } \\
\text { fzd5 } \\
\text { fzd8b } \\
\text { metap2b } \\
\text { rnf146 } \\
\text { tcf7l1b }\end{array}$ & $\begin{array}{l}\text { FGF signaling } \\
\text { fgfr1a } \\
\text { ext/3 } \\
\text { Epiboly } \\
\text { ezrb } \\
\text { sdc2 } \\
\text { Endoderm } \\
\text { dusp4 } \\
\text { Craniofacial dvp } \\
\text { furina }\end{array}$ & $\begin{array}{l}\text { Neurogenesis } \\
\text { IIgl1 } \\
\text { Wnt signaling } \\
f z d 7 b \\
\text { tcf7l1b } \\
\text { FGF signaling } \\
\text { fgfr1a } \\
\text { ext/3 } \\
\text { Epiboly } \\
\text { ezrb } \\
\text { Endoderm } \\
\text { dusp4 } \\
\text { Craniofacial dvp } \\
\text { furina }\end{array}$ & $\begin{array}{l}\text { Heart dvp } \\
\text { paf1 } \\
\text { adrbk2 } \\
\text { igf1rb } \\
\text { ilk } \\
\text { nfat5a } \\
\text { pkd2 } \\
\text { kctd10 } \\
\text { rarab } \\
\text { snai1b } \\
\text { tcf7l1b } \\
\text { D/V pattern } \\
\text { acvr2b }\end{array}$ \\
\hline
\end{tabular}

\section{Figure 5}


bioRxiv preprint doi: https://doi.org/10.1101/2021.08.26.457081; this version posted August 26, 2021. The copyright holder for this preprint

(which was not certified by peer review) is the author/funder, who has granted bioRxiv a license to display the preprint in perpetuity. It is made available under aCC-BY 4.0 International license.

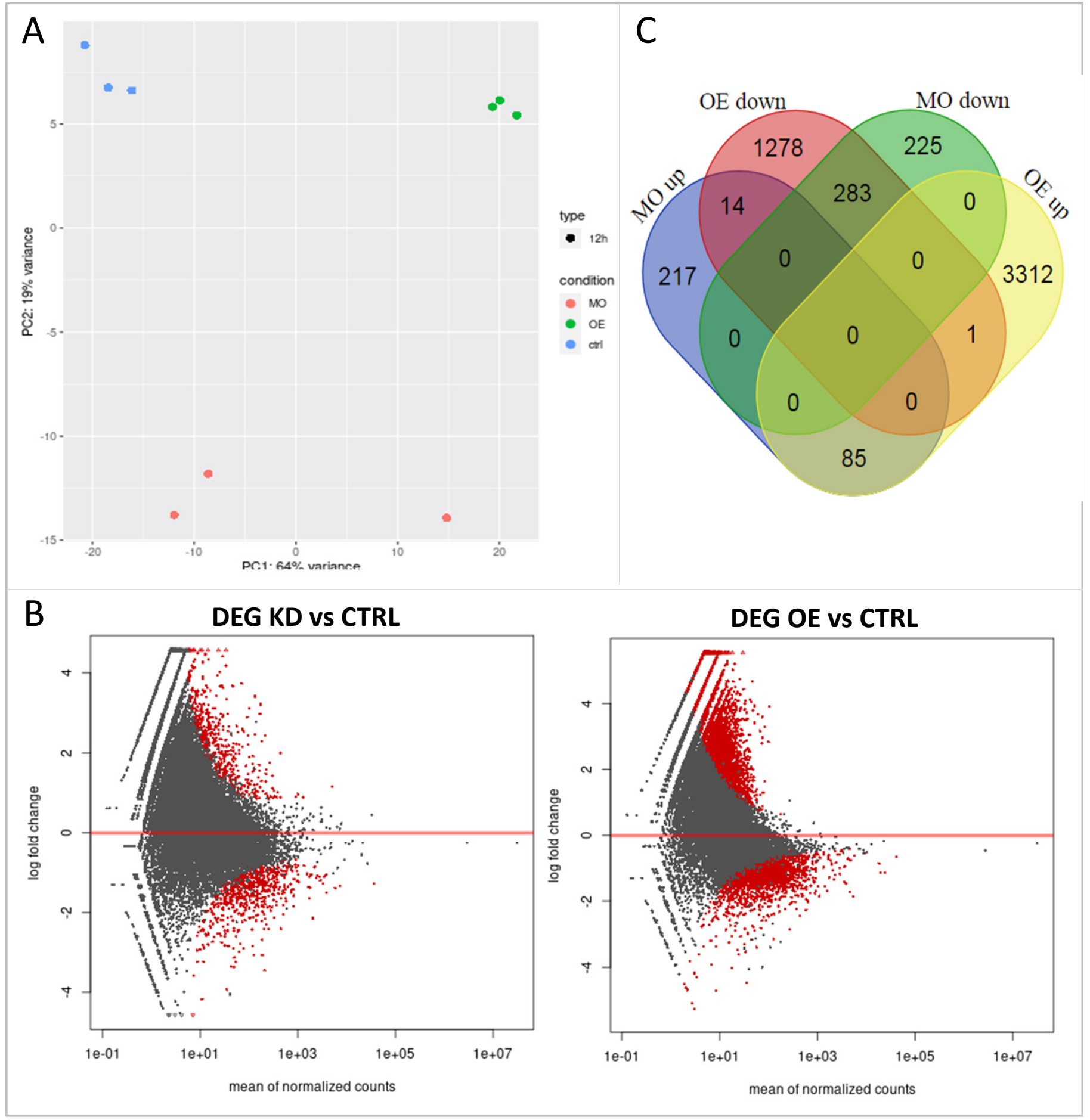

Figure 6 
bioRxiv preprint doi: https://doi.org/10.1101/2021.08.26.457081; this version posted August 26, 2021. The copyright holder for this preprint

(which was not certified by peer review) is the author/funder, who has granted bioRxiv a license to display the preprint in perpetuity. It is made available under aCC-BY 4.0 International license.

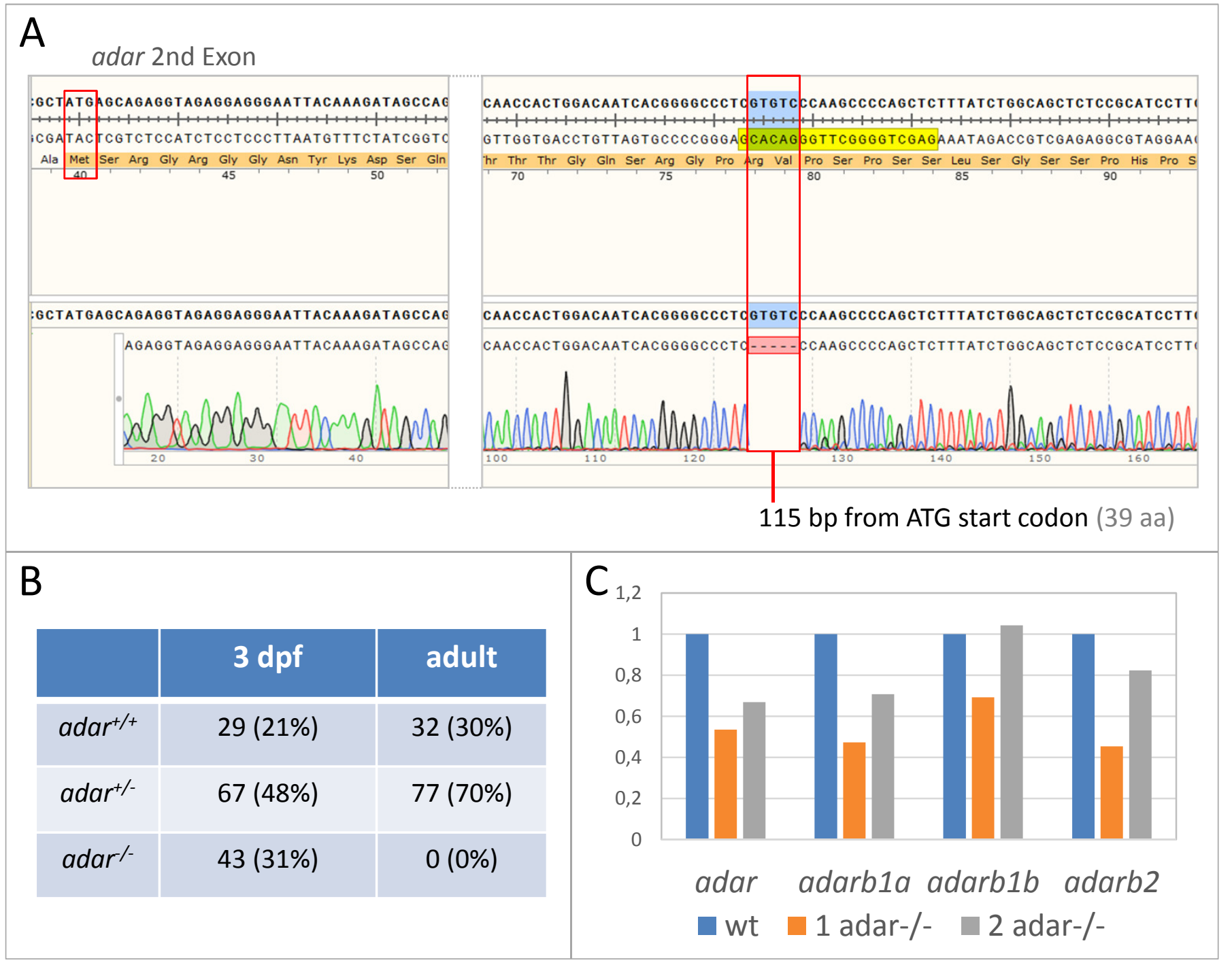

\section{Figure 7}




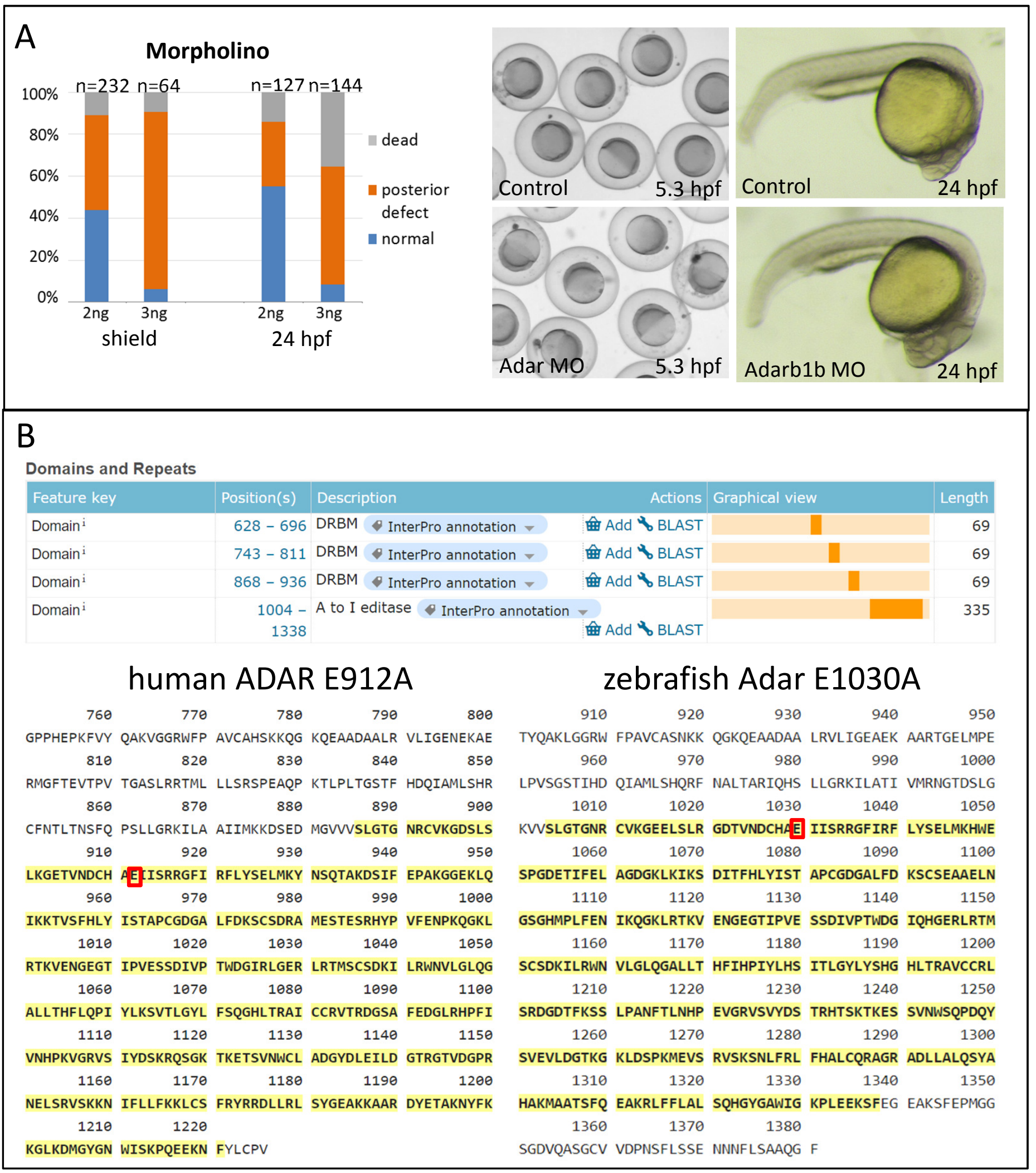

Supplementary Figure 1. Adar and Adarb1b knockdown experiments. (A) Dose- dependent effect of Adar MO was observed starting from 5.3 hpf, while Adarb1b MO did not cause any observable phenotype. (B) Design of adar mutant mRNA E1030A with point mutation abolishing the deaminase domain. 


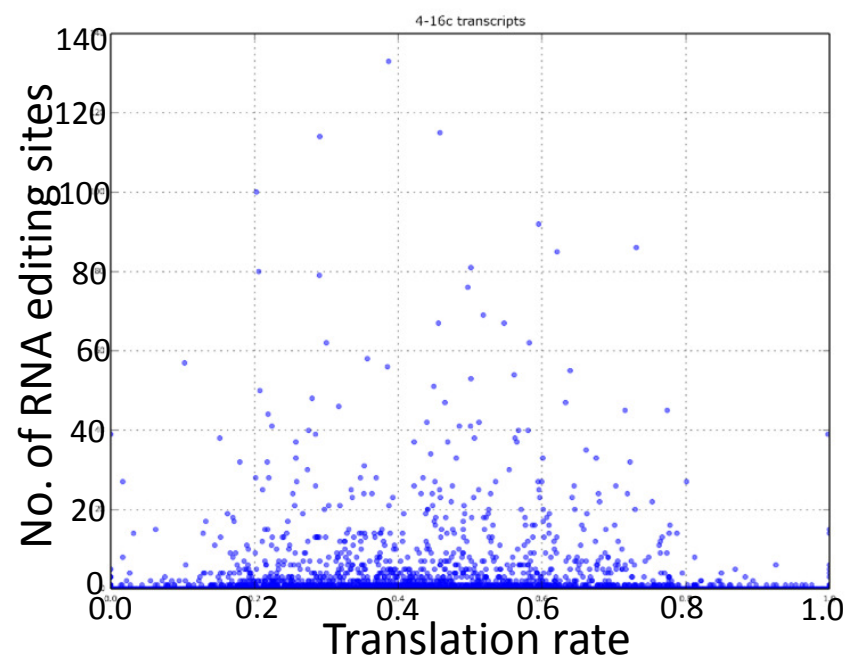
A.1.5 hpf
Pearson: $r=0.002 p=0.78$
Spearman: $r=0.016 p=0.04$

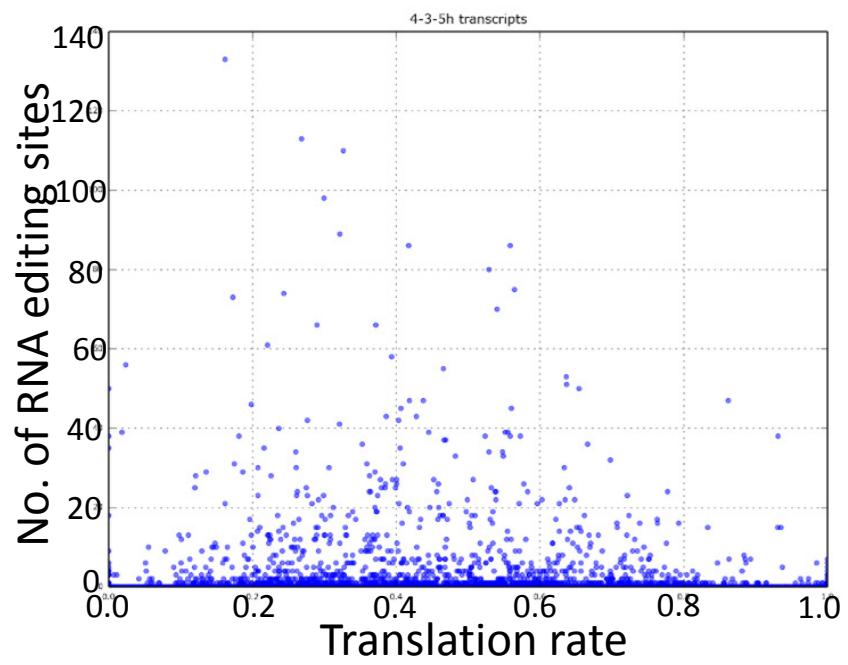

B. 3.5hpf

Pearson: $r=-0.053 p=3.79 e-11$

Spearman: $r=-0.077 p=2.36 e-22$

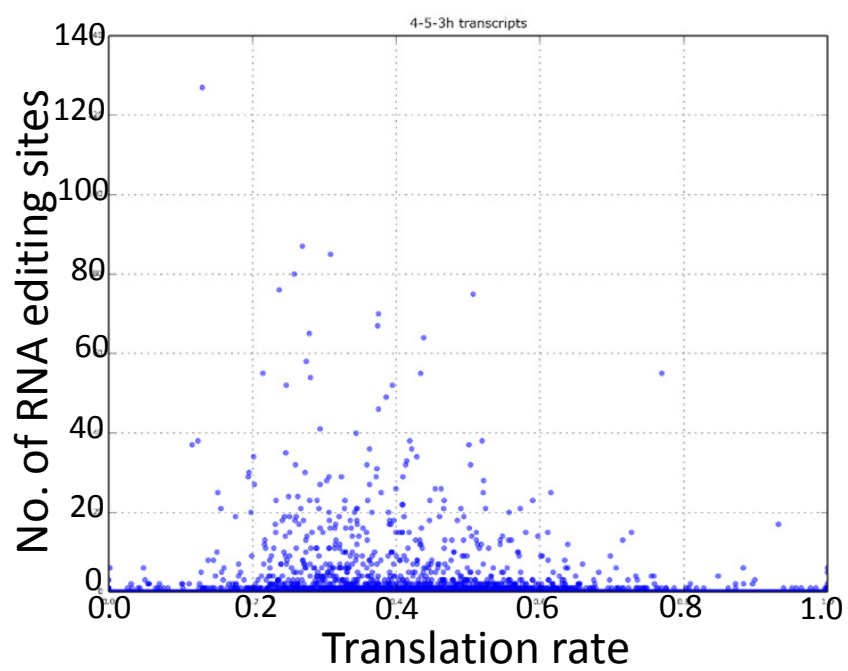

\section{C. $5.3 \mathrm{hpf}$}

Pearson: $r=-0.071 p=3.09 e-18$

Spearman: $r=-0.105 p=2.68 e-38$

Supplementary Figure 2. Translation rates and number of editing sites for $\mathbf{1 . 5} \mathrm{hpf}$ and $\mathbf{5 . 3} \mathrm{hpf}$ transcripts. Translation rates are expressed as ratio of polysome bound to sum of bound and unbound fractions of a given transcript (Winata et al., 2018). Thus, translation rate of 1.0 means all expressed transcript molecules are associated with polysome, while 0.0 means none of expressed transcript molecules are associated with polysome. 


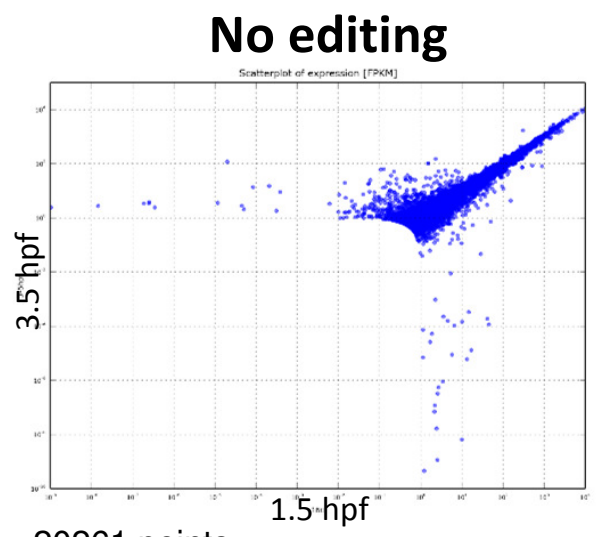

20261 points

Pearson: $r=0.989 p=0.0$

Spearman: $r=0.935 p=0.0$

Wilcoxon: $\mathrm{T}=98713441.5 \mathrm{p}=2.52 \mathrm{e}-06$

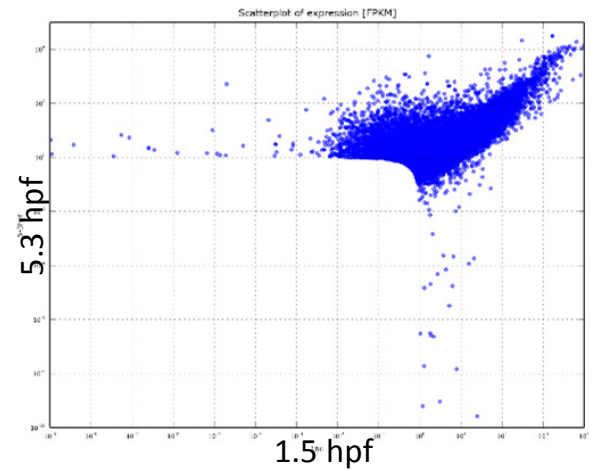

23940 points

Pearson: $r=0.683 p=0.0$

Spearman: $r=0.584 p=0.0$

Wilcoxon: $T=121005862.0 \mathrm{p}=2.02 \mathrm{e}-96$

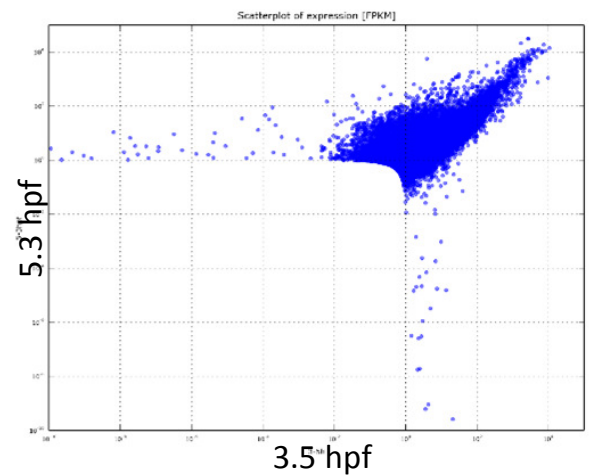

23920 points

Pearson: $r=0.734 p=0.0$

Spearman: $r=0.643 p=0.0$

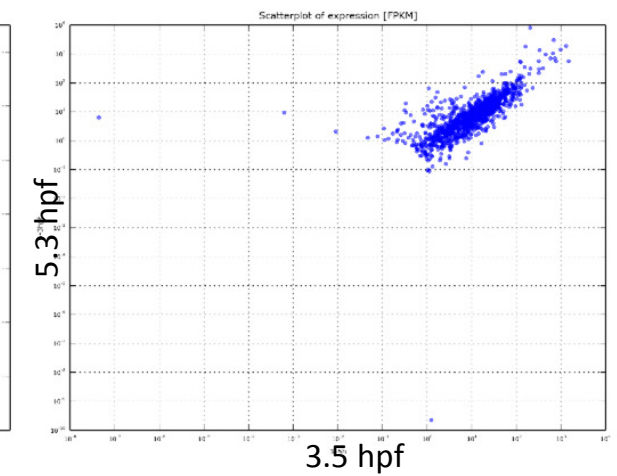

1171 points

Pearson: $r=0.439 p=1.75 e-56$

Spearman: $r=0.821 p=4.95 e-287$

Wilcoxon: $T=120227103.0 p=2.66 e-101$ Wilcoxon: $T=189785.0 p=4.79 e-40$
High editing ( $>10$ sites)

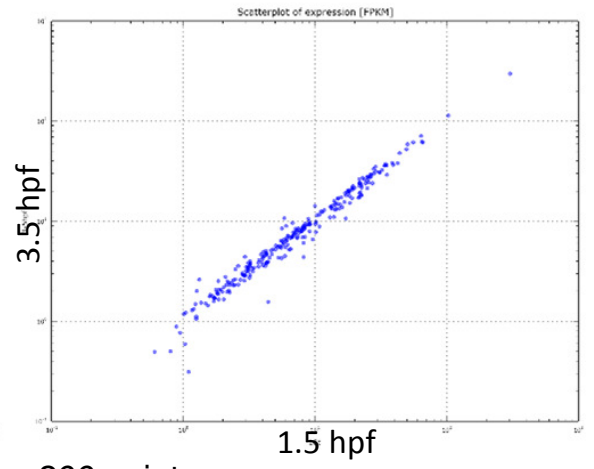

266 points

Pearson: $r=0.996 p=2.92 e-273$

Spearman: $r=0.976 p=1.27 e-176$

Wilcoxon: $\mathrm{T}=12841.0 \mathrm{p}=9.11 \mathrm{e}-05$

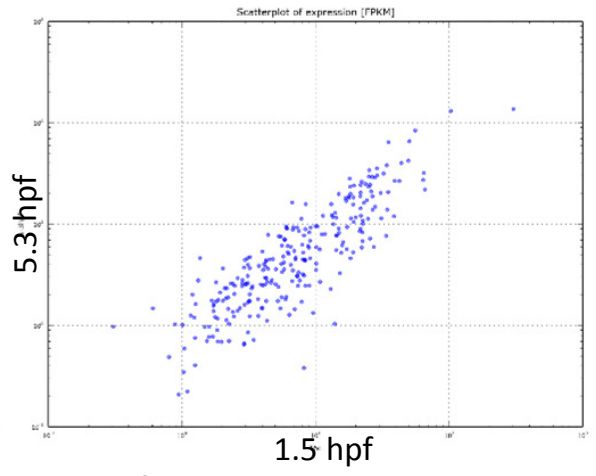

267 points

Pearson: $r=0.839 p=3.86 e-72$

Spearman: $r=0.846 p=2.31 e-74$

Wilcoxon: $T=7825.0 \mathrm{p}=1.61 \mathrm{e}-15$

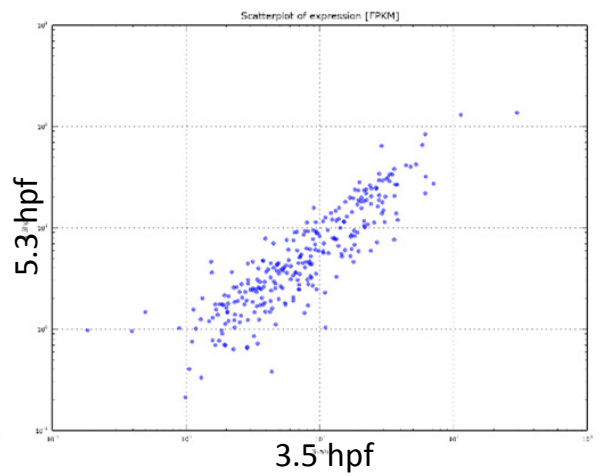

278 points

Pearson: $r=0.862 p=2.11 e-83$

Spearman: $r=0.889 p=1.69 \mathrm{e}-95$

Wilcoxon: $T=6651.0 p=2.2 e-21$

Supplementary Figure 3. Comparison of expression levels between two developmental stages of nonedited, low-edited, and highly edited transcripts. 

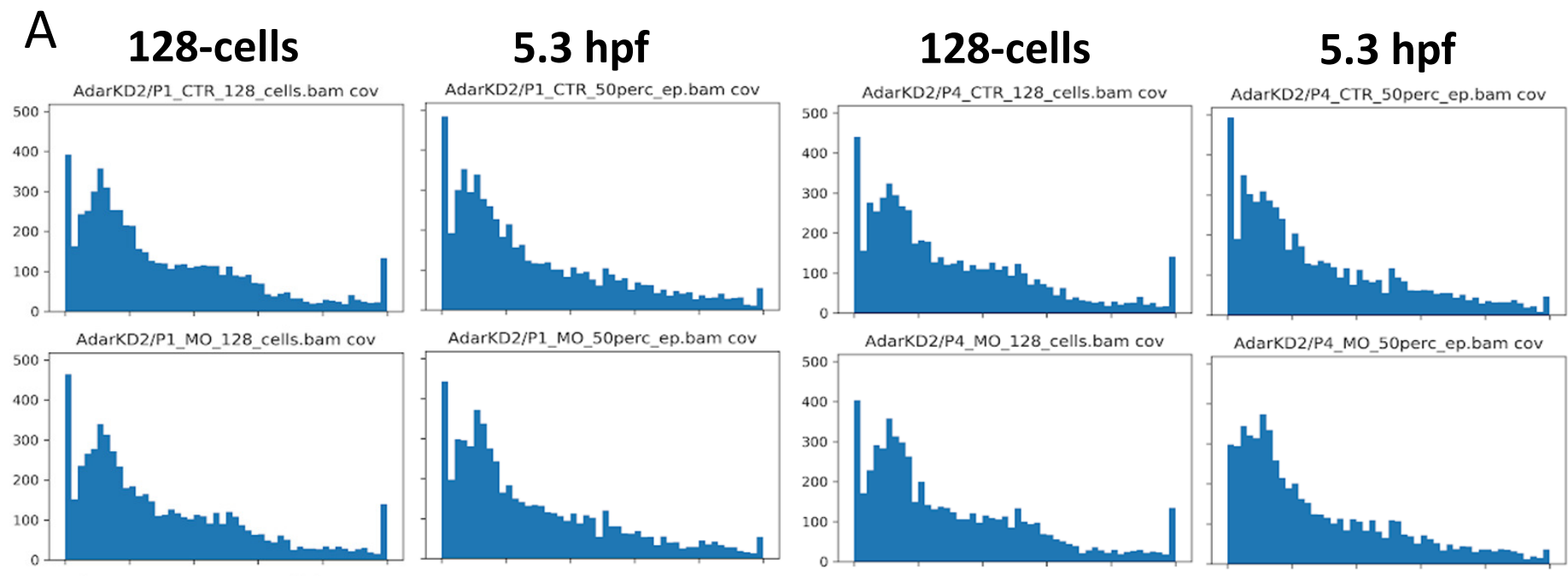

AdarKD2/P4_MO_50perc_ep.bam cov

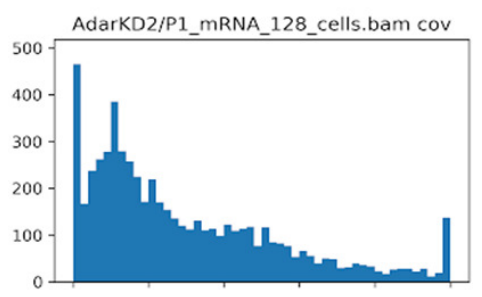

AdarKD2/P1_mRNA_50perc_ep.bam cov

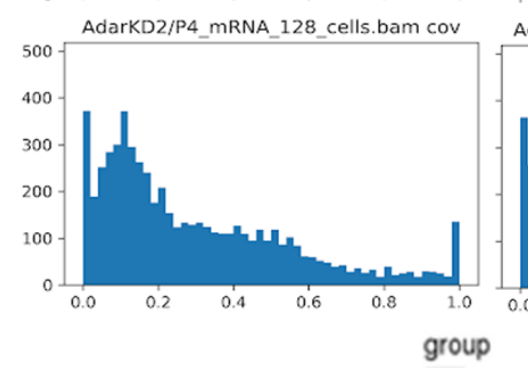

B
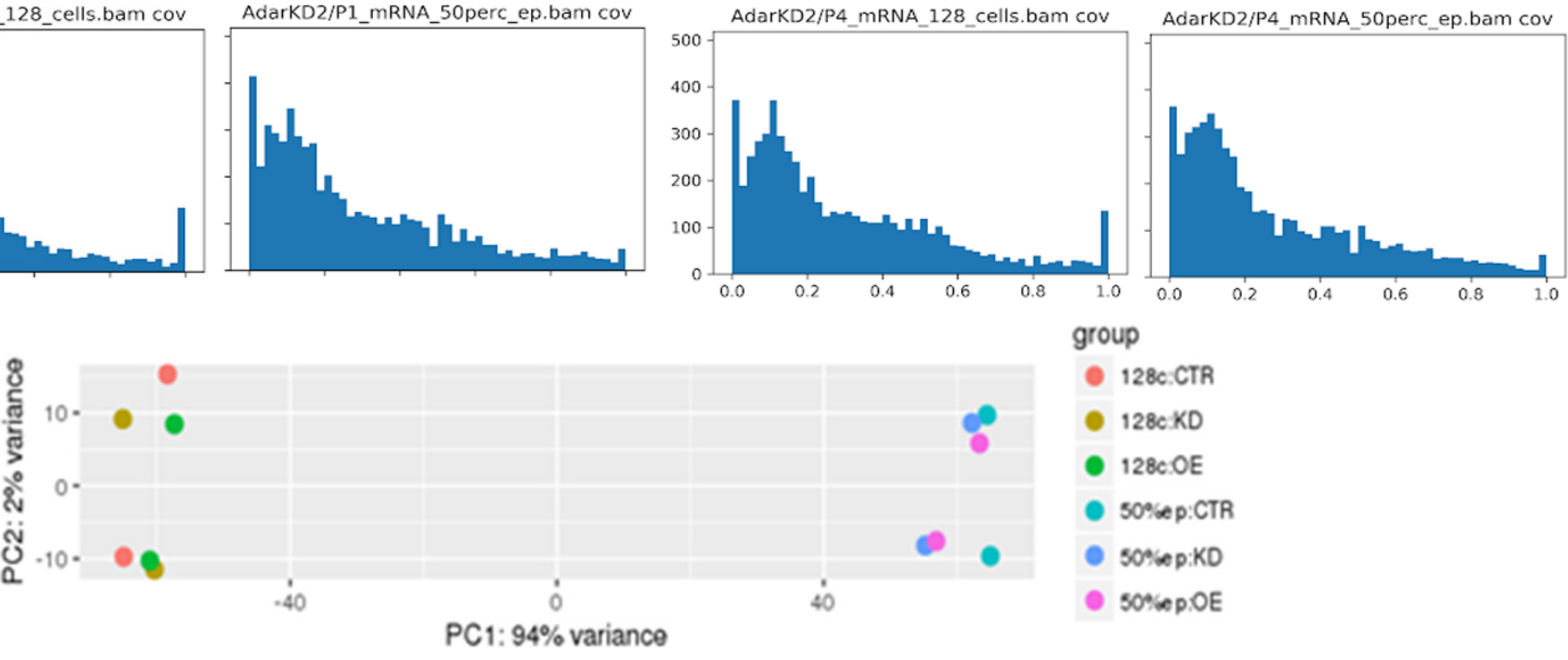

C

DEG KD vs CTR

DEG OE vs CTR
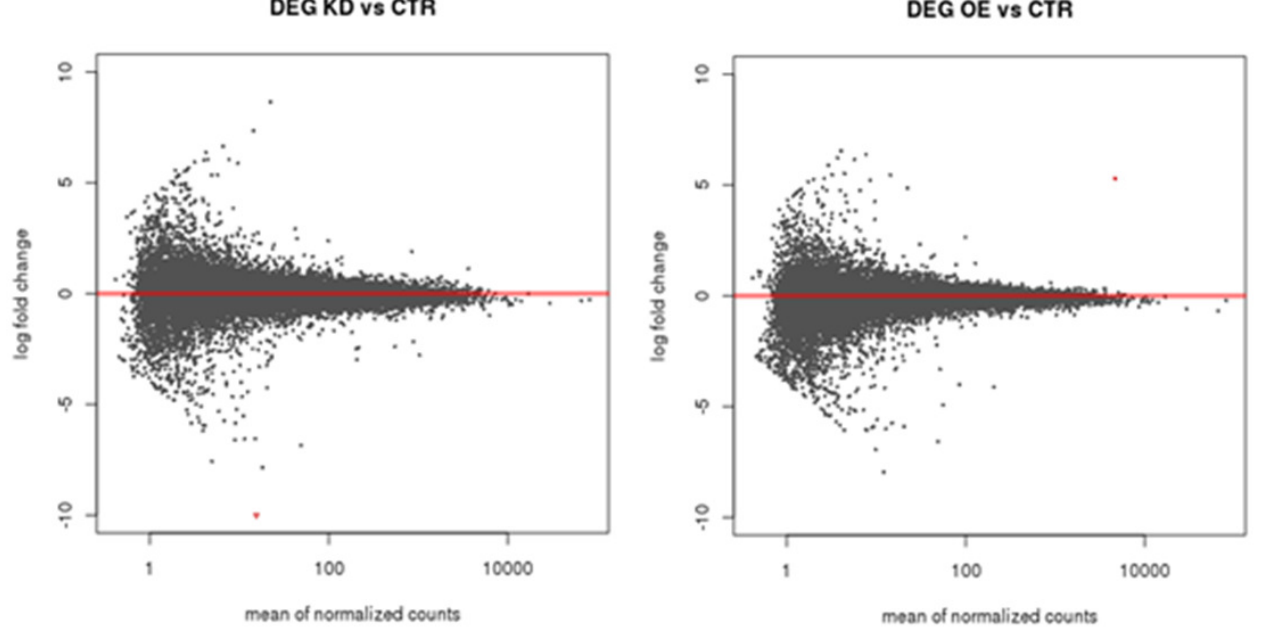

Supplementary Figure 4. Global RNA editing and gene expression profile of Adar knockdown and overexpression at 128-cell and 5.3 hpf. (A) Penetrance of RNA editing expressed as number of transcripts vs. fraction of editing within a transcript species in 128-cell and $5.3 \mathrm{hpf}$ stages. (B) Principal component analysis of control, Adar knockdown and overexpression samples based on their transcriptome profile. (C) Differential expression analysis of Adar knockdown and overexpression vs. control. No genes were differentially expressed at $p<0.05$. 
bioRxiv preprint doi: https://doi.org/10.1101/2021.08.26.457081; this version posted August 26, 2021. The copyright holder for this preprint (which was not certified by peer review) is the author/funder, who has granted bioRxiv a license to display the preprint in perpetuity. It is made available under aCC-BY 4.0 International license.
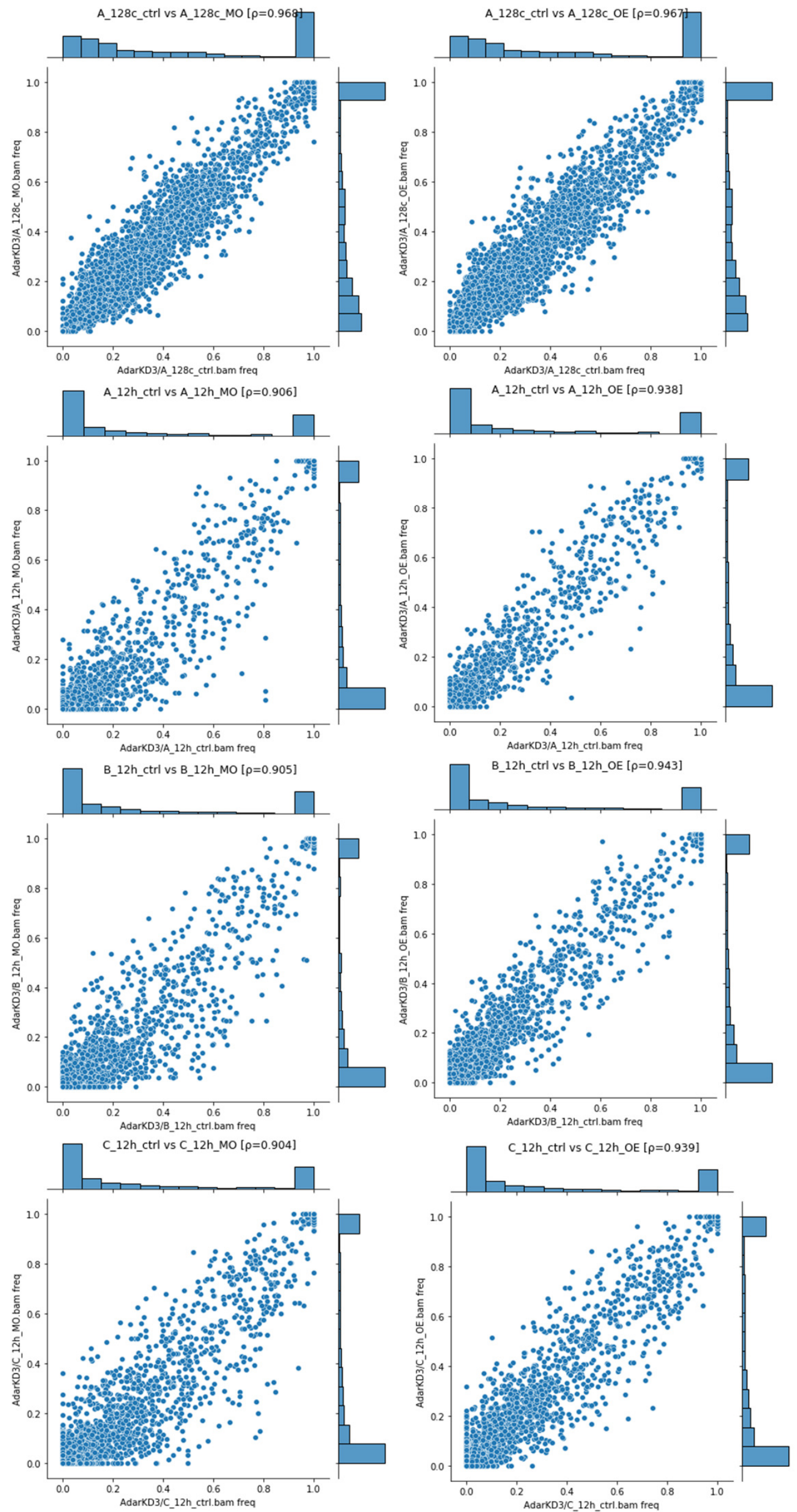

Supplementary Figure 5. Comparison of RNA editing frequency between control and Adar KD or OE. RNA editing frequencies for each transcript is plotted for each sample in 128-cell and 12 hpf stages. Spearman's rank correlation coefficient $(\rho)$ is given in every figure title. 